\title{
Known Algorithms on Graphs of Bounded Treewidth Are Probably Optimal
}

\author{
DANIEL LOKSHTANOV, Department of Informatics, University of Bergen \\ DÁNIEL MARX, Institute for Computer Science and Control, Hungarian Academy of Sciences \\ (MTA SZTAKI) \\ SAKET SAURABH, The Institute of Mathematical Sciences
}

We obtain a number of lower bounds on the running time of algorithms solving problems on graphs of bounded treewidth. We prove the results under the Strong Exponential Time Hypothesis of Impagliazzo and Paturi. In particular, assuming that $n$-variable $m$-clause SAT cannot be solved in time $(2-\epsilon)^{n} m^{O(1)}$, we show that for any $\epsilon>0$ :

- Independent Set cannot be solved in time $(2-\epsilon)^{\operatorname{tw}(G)}|V(G)|^{O(1)}$,

- Dominating Set cannot be solved in time $(3-\epsilon)^{\mathrm{tw}(G)}|V(G)|^{O(1)}$,

- Max Cut cannot be solved in time $(2-\epsilon)^{\operatorname{tw}(G)}|V(G)|^{O(1)}$,

- Odd Cycle Transversal cannot be solved in time $(3-\epsilon)^{\mathrm{tw}(G)}|V(G)|^{O(1)}$,

- For any fixed $q \geq 3, q$-Coloring cannot be solved in time $(q-\epsilon)^{\operatorname{tw}(G)}|V(G)| O(1)$,

- Partition Into Triangles cannot be solved in time $(2-\epsilon)^{\mathrm{tw}(G)}|V(G)|^{O(1)}$.

Our lower bounds match the running times for the best known algorithms for the problems, up to the $\epsilon$ in the base.

\section{CCS Concepts: • Theory of computation $\rightarrow$ Parameterized complexity and exact algorithms;}

Additional Key Words and Phrases: Treewidth, SETH, lower bounds

\section{ACM Reference format:}

Daniel Lokshtanov, Dániel Marx, and Saket Saurabh. 2018. Known Algorithms on Graphs of Bounded Treewidth Are Probably Optimal. ACM Trans. Algorithms 14, 2, Article 13 (April 2018), 30 pages.

https://doi.org/10.1145/3170442

A preliminary version of this aticle appeared in the proceedings of SODA 2011 [37].

Daniel Lokshtanov was supported by the Bergen Research Foundation and the University of Bergen through project "BeHard." Furthermore, he was supported by ERC Starting Grant PaPaAlg (No. 715744). Saket Saurabh was supported by the ERC Starting Grant PARAPPROX (No. 306992). Dániel Marx was supported by ERC Starting Grant PARAMTIGHT (No. 280152), and Consolidator Grant SYSTEMATICGRAPH (No. 755978).

Authors' addresses: D. Lokshtanov, Department of Informatics, University of Bergen, Norway, PB 7803, N-5020 Bergen; email: daniello@ii.uib.no; D. Marx, Institute for Computer Science and Control, Hungarian Academy of Sciences (MTA SZTAKI), P.O.B. 63, H-1518 Budapest, Hungary; email: dmarx@cs.bme.hu; S. Saurabh, Institute of Mathematical Sciences, CIT Campus, Taramani, Chennai, 600 113, India; email: saket@imsc.res.in.

Permission to make digital or hard copies of all or part of this work for personal or classroom use is granted without fee provided that copies are not made or distributed for profit or commercial advantage and that copies bear this notice and the full citation on the first page. Copyrights for components of this work owned by others than ACM must be honored. Abstracting with credit is permitted. To copy otherwise, or republish, to post on servers or to redistribute to lists, requires prior specific permission and/or a fee. Request permissions from permissions@acm.org.

(c) 2018 ACM 1549-6325/2018/04-ART13 $\$ 15.00$

https://doi.org/10.1145/3170442 


\section{INTRODUCTION}

It is well-known that many NP-hard graph problems can be solved efficiently if the treewidth $(\mathbf{t w}(G))$ of the input graph $G$ is bounded. For an example, an expository algorithm to solve VERTEX Cover and IndEPENDENT SET running in time $O^{*}\left(4^{\mathbf{t w}(G)}\right)$ is described in the algorithms textbook by Kleinberg and Tardos [35] (the $O^{*}$ notation suppresses factors polynomial in the input size), while the book by Niedermeier [43] on fixed-parameter algorithms presents an algorithm with running time $O^{*}\left(2^{\mathbf{t w}(G)}\right)$. Similar algorithms, with running times on the form $O^{*}\left(c^{\mathbf{t w}(G)}\right)$ for a constant $c$, are known for many other graph problems such as Dominating SET, $q$-Coloring, and Odd Cycle Transversal [5, 19, 26, 27, 50]. Algorithms for graph problems on bounded treewidth graphs have found many uses as subroutines in approximation algorithms [7, 24, 25, 36], parameterized algorithms [4, 21, 23, 34, 41, 49], and exact algorithms [28, 42, 46].

In this article, we show that any improvement over the currently best known algorithms for a number of well-studied problems on graphs of bounded treewidth would yield a faster algorithm for SAT. In particular, we show if there exists an $\epsilon>0$ such that

- Independent Set can be solved in time $O^{*}\left((2-\epsilon)^{\mathbf{t w}(G)}\right)$, or

- Dominating Set can be solved in time $O^{*}\left((3-\epsilon)^{\mathbf{t w}(G)}\right)$, or

- Max Cut can be solved in time $O^{*}\left((2-\epsilon)^{\mathbf{t} \mathbf{w}(G)}\right)$, or

- Odd Cycle Transversal can be solved in time $O^{*}\left((3-\epsilon)^{\mathbf{t w}(G)}\right)$, or

- there is a fixed $q \geq 3$ such that $q$-Coloring can be solved in time $O^{*}\left((q-\epsilon)^{\mathbf{t} \mathbf{w}(G)}\right)$, or

- Partition Into Triangles can be solved in time $O^{*}\left((2-\epsilon)^{\mathbf{t w}(G)}\right)$,

then $n$-variable SAT can be solved in $O^{*}\left((2-\delta)^{n}\right)$ time for some $\delta>0$. Such an algorithm would violate the Strong Exponential Time Hypothesis (SETH) of Impagliazzo and Paturi [31]. Thus, assuming SETH, the known algorithms for the mentioned problems on graphs of bounded treewidth are essentially the best possible.

To show our results, we give polynomial time many-one reductions that transform $n$-variable boolean formulas $\phi$ to instances of the problems in question. Such reductions are well-known, but for our results, we need to carefully control the treewidth of the graphs that our reductions output. A typical reduction creates $n$ gadgets corresponding to the $n$ variables; each gadget has a small constant number of vertices. In most cases, this implies that the treewidth can be bounded by $O(n)$. However, to prove the lower bound of the form $O^{*}\left((2-\epsilon)^{\mathbf{t w}(G)}\right)$, we need that the treewidth of the constructed graph is $(1+o(1)) n$. Thus, we can afford to increase the treewidth by at most one per variable. For lower bounds above $O^{*}\left((2-\epsilon)^{\mathbf{t w}(G)}\right)$, we need even more economical constructions. To understand the difficulty, consider the Dominating SET problem; here, we want to say that if Dominating SET admits an algorithm with running time $O^{*}\left((3-\epsilon)^{\mathbf{t w}(G)}\right)=O^{*}\left(2^{\log (3-\epsilon) \mathbf{t w}(G)}\right)$ for some $\epsilon>0$, then we can solve SAT on input formulas with $n$-variables in time $O^{*}\left((2-\delta)^{n}\right)$ for some $\delta>0$. Therefore, by naïvely equating the exponent in the previous sentence, we get that we need to construct an instance for Dominating SET whose treewidth is essentially $\frac{n}{\log 3}$. In other words, each variable should increase treewidth by less than one. The main challenge in our reductions is to squeeze out as many combinatorial possibilities per increase of treewidth as possible. To control the treewidth of the graphs we construct, we upper bound the pathwidth $(\mathbf{p w}(G))$ of the constructed instances and use the fact that for any graph $G, \mathbf{t w}(G) \leq \mathbf{p w}(G)$. Thus, all of our lower bounds also hold for problems on graphs of bounded pathwidth.

Complexity Assumption: The Exponential Time Hypothesis (ETH) and its strong variant (SETH) are conjectures about the exponential time complexity of $k$-SAT. The $k$-SAT problem is a restriction of SAT, where every clause in input boolean formula $\phi$ has at most $k$ literals. Let $s_{k}=\inf \{\delta: k$-SAT 
can be solved in $2^{\delta n}$ time\}. The Exponential Time Hypothesis conjectured by Impagliazzo, Paturi, and Zane [32] is that $s_{3}>0$. In Reference [32], it is shown that ETH is robust, that is $s_{3}>0$ if and only if there is a $k \geq 3$ such that $s_{k}>0$. In the same year, it was shown that assuming ETH the sequence $\left\{s_{k}\right\}$ increases infinitely often [31]. Since SAT has a $O^{*}\left(2^{n}\right)$ time algorithm, $\left\{s_{k}\right\}$ is bounded by above by one, and Impagliazzo and Paturi [31] conjecture that 1 is indeed the limit of this sequence. In a subsequent article [12], this conjecture is coined as SETH.

ETH is now a widely believed assumption and has been used as a starting point to prove running time lower bounds for numerous problems [9, 14, 15, 18, 22, 29, 38-40]. At the time of the conference version of this article, SETH was largely untouched [11, 16, 17, 20, 21, 44]. The reason for this was twofold. First, the assumption that $\lim _{k \rightarrow \infty} s_{k}=1$ is a very strong one. Second, when proving lower bounds under ETH, we can utilize the Sparsification Lemma [32], which allows us to reduce from instances of 3-SAT where the number of clauses is linear in the number of variables. Such a tool does not exist for SETH, and this seems to be a major obstruction for showing running time lower bounds for interesting problems under SETH. We overcome this obstruction by circumventing it-to show running time lower bounds for algorithms on bounded treewidth graphs sparsification is simply not required.

Related Work. In several cases designing the "right algorithm" on graphs of bounded treewidth or pathwidth is not at all obvious. For example: Alber et al. [5] gave a $O^{*}\left(4^{\mathbf{t w}(G)}\right)$ time algorithm for Dominating SET, improving over the natural $O^{*}\left(9^{\mathbf{t w}(G)}\right)$ algorithm of Telle and Proskurowski [48]. Later, van Rooij et al. [50] observed that one could use fast subset convolution [8] to improve the running time of algorithms on graphs of bounded treewidth. Their results include a $O^{*}\left(3^{\mathbf{t w}(G)}\right)$ algorithm for Dominating SET and a $O^{*}\left(2^{\mathbf{t w}(G)}\right)$ time algorithm for PARtition Into Triangles. Interestingly, the effect of applying subset convolution was that the running time for several graph problems on bounded treewidth graphs became the same as the running time for the problems on graphs of bounded pathwidth. However, the idea of using subset convolution in designing dynamic programming algorithm over graphs of bounded treewidth was not enough to design "optimal algorithms" for several connectivity problems such as Hamiltonian Path and Connected Vertex Cover. In a seminal article, Cygan et al. [21] introduced the method of Cut \& Count and designed the first $O^{*}\left(c^{\mathbf{t w}(G)}\right)$, where $c$ is a fixed constant, for plethora of connectivity problems including Hamiltonian Path and Connected Vertex Cover. However, the algorithm for Hamiltonian PATH runs in time $O^{*}\left(4^{\mathbf{t w}(G)}\right)$, which still is the best known algorithm. Later, in a surprising result, Cygan et al. [20] showed that Hamiltonian Path can be solved in time $O^{*}\left((2+\sqrt{2})^{\mathbf{p w}(G)}\right)$ on graphs of bounded pathwidth. The algorithms obtained using Cut \& Count are randomized. Later, deterministic algorithms with running time $O^{*}\left(c^{\mathbf{t w}(G)}\right)$, where $c$ is a fixed constant, were designed for connectivity problems [10,30].

Follow-up Work. The problems considered in this article, and the ideas used to resolve them, led to several follow-up works that showed lower bounds for concrete problems in the parameterized settings [11, 16, 17, 21]. On the other hand, Roditty and Williams [45] used SETH to show concrete lower bounds on the running time for problems solvable in polynomial time. This was followed by a long line of work in this direction [1-3, 6, 13, 51]. The work of Cygan et al. [21] that introduced the method of Cut \& Count to design $O^{*}\left(c^{\mathbf{t w}(G)}\right)$, where $c$ is a fixed constant, for connectivity problems, also showed that the base of exponent in their algorithm are optimal unless SETH fails. Cygan et al. [20] showed that the running time of $O^{*}((2+\sqrt{2}) \mathbf{p w}(G))$ for Hamiltonian Path on graphs of bounded pathwidth is, in fact, optimal under SETH. Several other lower bounds for concrete problems were also obtained in Reference [17]. Ideas from the current article were recently 
used to design tight lower bounds for $r$-Dominating Set and Connected Dominating Set on graphs of bounded treewidth [11]. Curticapean and Marx obtained tight lower bounds for counting perfect matchings on graphs of bounded treewidth, cliquewidth, and genus under SETH [16].

\section{PRELIMINARIES}

In this section, we give various definitions that we make use of in the article. Let $G$ be a graph with vertex set $V(G)$ and edge set $E(G)$. A graph $G^{\prime}$ is a subgraph of $G$ if $V\left(G^{\prime}\right) \subseteq V(G)$ and $E\left(G^{\prime}\right) \subseteq$ $E(G)$. For a subset $V^{\prime} \subseteq V(G)$, the subgraph $G^{\prime}=G\left[V^{\prime}\right]$ of $G$ is called a subgraph induced by $V^{\prime}$ if $E\left(G^{\prime}\right)=\left\{u v \in E(G) \mid u, v \in V^{\prime}\right\}$ and $V\left(G^{\prime}\right)=V^{\prime}$. By $N(u)$, we denote the (open) neighborhood of $u$ in graph $G$, that is, the set of all vertices adjacent to $u$ and by $N[u]=N(u) \cup\{u\}$. Similarly, for a subset $D \subseteq V(G)$, we define $N[D]=\cup_{v \in D} N[v]$.

A tree decomposition of a graph $G$ is a pair $(\mathcal{X}, T)$, where $T$ is a tree and $\mathcal{X}=\left\{X_{i} \mid i \in V(T)\right\}$ is a collection of subsets of $V(G)$ such that: 1. $\bigcup_{i \in V(T)} X_{i}=V(G)$, 2. for each edge $x y \in E(G)$, $\{x, y\} \subseteq X_{i}$ for some $i \in V(T)$; 3 . for each $x \in V(G)$ the set $\left\{i \mid x \in X_{i}\right\}$ induces a connected subtree of $T$. The width of the tree decomposition is $\max _{i \in V(T)}\left\{\left|X_{i}\right|-1\right\}$. The treewidth of a graph $G$ is the minimum width over all tree decompositions of $G$. We denote by $\mathbf{t w}(G)$ the treewidth of graph $G$. If in the definition of treewidth we restrict the tree $T$ to be a path, then we get the notion of pathwidth and denote it by $\mathbf{p w}(G)$. For our purpose, we need an equivalent definition of pathwidth via mixed search games.

In a mixed search game, a graph $G$ is considered as a system of tunnels. Initially, all edges are contaminated by a gas. An edge is cleared by placing searchers at both its end-points simultaneously or by sliding a searcher along the edge. A cleared edge is re-contaminated if there is a path from an uncleared edge to the cleared edge without any searchers on its vertices or edges. A search is a sequence of operations that can be of the following types: (a) placement of a new searcher on a vertex; (b) removal of a searcher from a vertex; (c) sliding a searcher on a vertex along an incident edge and placing the searcher on the other end. A search strategy is winning if after its termination all edges are cleared. The mixed search number of a graph $\mathrm{G}$, denoted by $\mathbf{m s}(G)$, is the minimum number of searchers required for a winning strategy of mixed searching on $G$. Takahashi, Ueno, and Kajitani [47] obtained the following relationship between $\mathbf{p w}(G)$ and $\mathbf{m s}(G)$, which we use for bounding the pathwidth of the graphs obtained in reduction.

Proposition 1 ([47]). For a $\operatorname{graph} G, \mathbf{p w}(G) \leq \mathbf{m s}(G) \leq \mathbf{p w}(G)+1$.

An instance to SAT consists of a boolean formula $\phi=C_{1} \wedge \cdots \wedge C_{m}$ over $n$ variables $\left\{v_{1}, \ldots, v_{n}\right\}$, where each clause $C_{i}$ is OR of one or more literals of variables. We also denote a clause $C_{i}$ by the set $\left\{\ell_{1}, \ell_{2}, \ldots, \ell_{c}\right\}$ of its literals and denote by $\left|C_{i}\right|$ the number of literals in $C_{i}$. An assignment $\tau$ to the variables is an element of $\{0,1\}^{n}$, and it satisfies the formula $\phi$ if for every clause $C_{i}$ there is literal that is assigned 1 by $\tau$. We say that a variable $v_{i}$ satisfies a clause $C_{j}$ if there exists a literal corresponding to $v_{i}$ in $\left\{\ell_{1}, \ell_{2}, \ldots, \ell_{c}\right\}$ and it is set to 1 by $\tau$. A group of variables satisfy a clause $C_{j}$ if there is a variable that satisfies the clause $C_{j}$. All the sections in this article follow the same pattern: definition of the problem; statement of the lower bound; construction used in the reduction; correctness of the reduction; and the upper bound on the pathwidth of the resultant graph.

\section{INDEPENDENT SET}

An independent set of a graph $G$ is a set $S \subseteq V(G)$ such that $G[S]$ contains no edges. In the INDEPENDENT SET problem, we are given a graph $G$ and the objective is to find an independent set of maximum size. 
We first sketch the main idea of the proof. We give the reduction from an arbitrary SAT instance on $n$ variables and $m$ clauses. The idea is to create a family of $n$ very long paths $P_{1}, P_{2}, \ldots, P_{n}$ of even length, corresponding to variables $x_{1}, x_{2}, \ldots, x_{n}$. Assume for now that on each of these paths the solution is allowed to make one of two choices: the independent set either contains all the odd-indexed vertices or all the even-indexed vertices. Then, for every clause we construct a clause verification gadget and attach it to some place in the family. The gadget is adjacent to paths corresponding to variables appearing in the clause, and the attachment points reflect whether the variable's appearance is positive or negative. The role of the clause gadget is to verify that the clause is satisfied. Satisfaction of the clause corresponds to the condition that at least one of the attachment points of the clause gadget needs to be not chosen into the constructed independent set; hence, the clause gadget needs to have the following property: the behavior inside the gadget can be set optimally if and only if at least one of the attachment points is free. It is possible to construct a gadget with exactly this property, and moreover the gadget has constant pathwidth, so it does not increase much the width of the whole construction. One technical problem that we still need to overcome is the first technical assumption about the choices the solution makes on the paths $P_{i}$. It is namely not true that on a path of even length there are only two maximum-size independent sets: the odd-indexed vertices and the even-indexed vertices. The solution can first start with picking only odd-indexed vertices, then make a gap of two vertices, and continue further with even-indexed vertices. Thus, on each path there can be one "cheat" where the solution flips from odd indices to even indices. The solution to this problem is a remarkably simple trick that is commonly used in similar reductions. We namely repeat the whole sequence of clause gadgets $n+1$ times, which ensures that at most $n$ copies are spoiled by possible cheats, and hence at least one of the copies is attached to an area where no cheat happens, and hence the behavior of the solution on the paths $P_{i}$ correctly encodes some satisfying assignment of the variable set. This concludes the sketch, and we move toward giving the formal proof.

TheOREM 1. If INDEPENDENT SET can be solved in $O^{*}\left((2-\epsilon)^{\mathbf{t w}(G)}\right)$ for some $\epsilon>0$, then SAT can be solved in $O^{*}\left((2-\delta)^{n}\right)$ time for some $\delta>0$.

Construction. Given an instance $\phi$ of SAT, we construct a graph $G$ as follows (see Figure 1). We assume that every clause has an even number of variables: if not, we can add a single variable to all odd size clauses and force this variable to false. First, we describe the construction of clause gadgets. For a clause $C=\left\{\ell_{1}, \ell_{2}, \ldots, \ell_{c}\right\}$, we introduce a gadget $\widehat{C}$ as follows. We take two paths, $C P=c p_{1}, c p_{2} \ldots, c p_{c}$ and $C P^{\prime}=c p_{1}^{\prime}, c p_{2}^{\prime} \ldots c p_{c}^{\prime}$ having $c$ vertices each, and connect $c p_{i}$ with $c p_{i}^{\prime}$ for every $i$. For each literal $\ell_{i}$, we introduce a vertex $\ell_{i}$ in $\widehat{C}$ and make it adjacent to $c p_{i}$ and $c p_{i}^{\prime}$. Finally, we add two vertices $c_{\text {start }}$ and $c_{\text {end }}$, such that $c_{\text {start }}$ is adjacent to $c p_{1}$ and $c_{\text {end }}$ is adjacent to $c p_{c}$. Observe that the size of the maximum independent set of $\widehat{C}$ is $c+2$. Also, since $c$ is even, any independent set of size $c+2$ in $\widehat{C}$ must contain at least one vertex in $C=\left\{\ell_{1}, \ell_{2}, \ldots, \ell_{c}\right\}$. Finally, notice that for any $i$, there is an independent set of size $c+2$ in $\widehat{C}$ that contains $\ell_{i}$ and none of $\ell_{j}$ for $j \neq i$.

We first construct a graph $G_{1}$. We introduce $n$ paths $P_{1}, \ldots, P_{n}$, each path has $2 m$ vertices. Let the vertices of the path $P_{i}$ be $p_{i}^{1} \ldots p_{i}^{2 m}$. The path $P_{i}$ corresponds to the variable $v_{i}$. For every clause $C_{i}$ of $\phi$, we introduce a gadget $\widehat{C}_{i}$. Now, for every variable $v_{i}$, if $v_{i}$ occurs positively in $C_{j}$, we add an edge between $p_{i}^{2 j}$ and the literal corresponding to $v_{i}$ in $\widehat{C}_{j}$. If $v_{i}$ occurs negatively in $C_{j}$, then we add an edge between $p_{i}^{2 j-1}$ and the literal corresponding to $v_{i}$ in $\widehat{C}_{j}$. Now, we construct the graph $G$ as follows. We take $n+1$ copies of $G_{1}$, call them $G_{1}, \ldots, G_{n+1}$. For every $i \leq n$, we connect $G_{i}$ and $G_{i+1}$ by connecting $p_{j}^{2 m}$ in $G_{i}$ with $p_{j}^{1}$ in $G_{i+1}$ for every $j \leq n$. This way, the paths $P_{j}$ in each of 


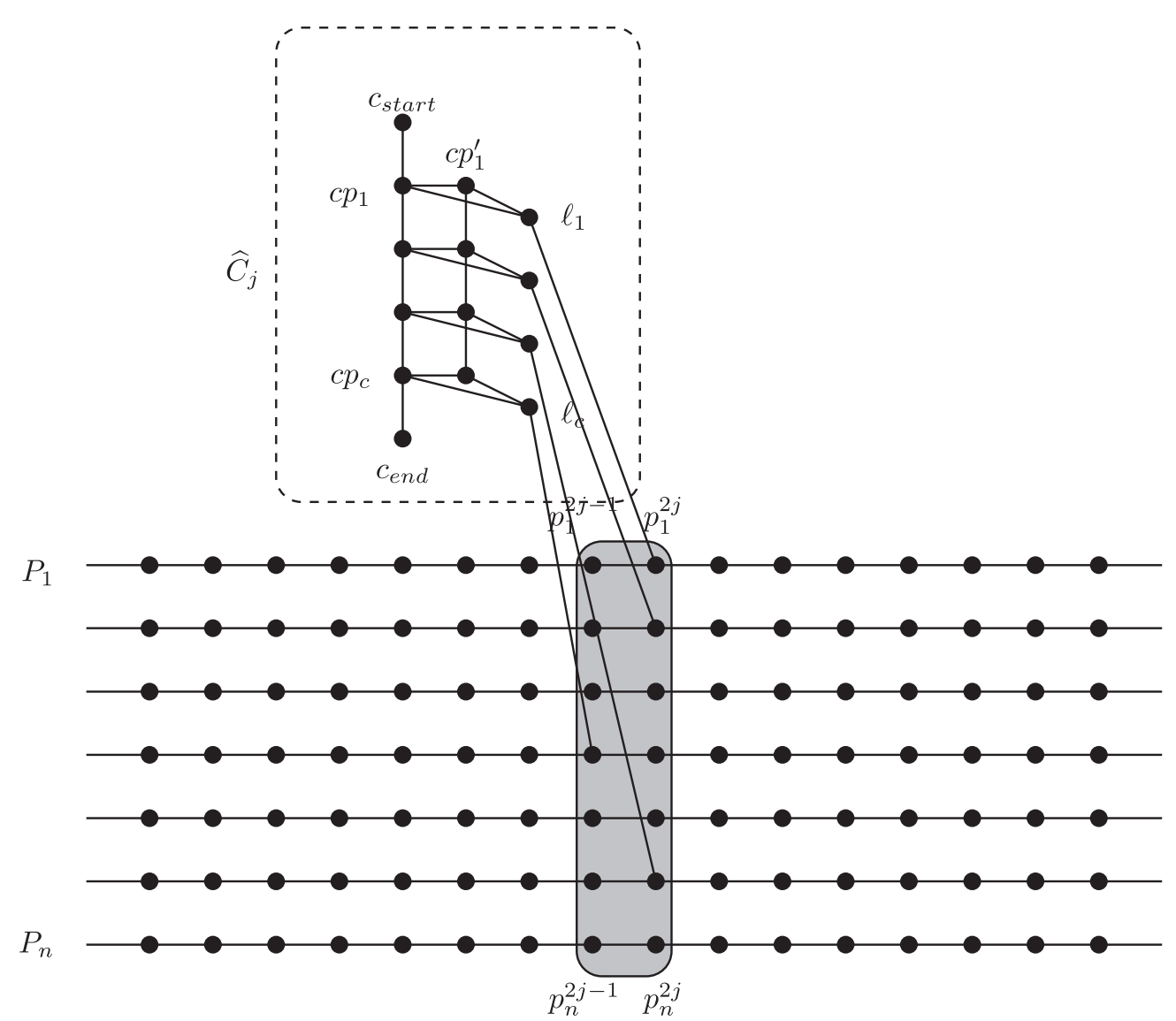

Fig. 1. Reduction to INDEPENDENT SET: clause gadget $\widehat{C}_{j}$ attached to the $n$ paths representing the variables.

the $n$ copies $G_{i}$ together form a long path of $2 m(n+1)$ vertices. This concludes the construction of $G$.

Lemma 1. If $\phi$ is satisfiable, then $G$ has an independent set of size $\left(m n+\sum_{i \leq m}\left(\left|C_{i}\right|+2\right)\right)(n+1)$.

Proof. Consider a satisfying assignment to $\phi$. We construct an independent set $I$ in $G$. For every variable $v_{i}$, if $v_{i}$ is set to true, then pick all the vertices on odd positions from all copies of $P_{i}$, that is $p_{i}^{1}, p_{i}^{3}, p_{i}^{5}$ and so on. If $v_{i}$ is false, then pick all the vertices on even positions from all copies of $P_{i}$, that is $p_{i}^{2}, p_{i}^{4}, p_{i}^{6}$ and so on. It is easy to see that this is an independent set of size $m n(n+1)$ containing vertices from all the paths. We will now consider the gadget $\widehat{C}_{j}$ corresponding to a clause $C_{j}$. We will only consider the copy of $\widehat{C}_{j}$ in $G_{1}$ as the other copies can be dealt identically. Let us choose a true literal $\ell_{a}$ in $C_{j}$ and let $v_{i}$ be the corresponding variable. Consider the vertex $\ell_{a}$ in $\widehat{C}_{j}$. If $v_{i}$ occurs positively in $C_{j}$, then $v_{i}$ is true. Then $I$ does not contain $p_{i}^{2 j}$, the only neighbour of $\ell_{a}$ outside of $\widehat{C}_{j}$. On the other hand, if $v_{i}$ occurs negatively in $C_{j}$, then $v_{i}$ is false. In this case $I$ does not contain $p_{i}^{2 j-1}$, the only neighbour of $\ell_{a}$ outside of $\widehat{C}_{j}$. There is an independent set of size $\left|C_{j}\right|+2$ in $\widehat{C}$ that contains $\ell_{a}$ and none out of $\ell_{b}$ for any $b \neq a$. We add this independent set to $I$ and proceed in this manner for every clause gadget. By the end of the process $\left(\sum_{i \leq m}\left(\left|C_{i}\right|+2\right)\right)(n+1)$, vertices 
from clause gadgets are added to $I$, yielding that the size of $I$ is $\left(m n+\sum_{i \leq m}\left(\left|C_{i}\right|+2\right)\right)(n+1)$, concluding the proof.

Lemma 2. If $G$ has an independent set of size $\left(m n+\sum_{i \leq m}\left(\left|C_{i}\right|+2\right)\right)(n+1)$, then $\phi$ is satisfiable.

Proof. Consider an independent set of $G$ of size $\left(m n+\sum_{i \leq m}\left|C_{i}\right|+2\right)(n+1)$. Set $I$ can contain at most $m$ vertices from each copy of $P_{i}$ for every $i \leq n$ and at most $\left|C_{j}\right|+2$ vertices from each copy of the gadget $C_{j}$. Since $I$ must contain at least that many vertices from each path and clause gadget in order to contain at least $\left(m n+\sum_{i \leq m}\left|C_{i}\right|+2\right)(n+1)$ vertices, it follows that $I$ has exactly $m$ vertices in each copy of each path $P_{i}$ and exactly $\left|C_{j}\right|+2$ vertices in each copy of each clause gadget $\widehat{C}_{j}$. For a fixed $j$, consider the $n+1$ copies of the path $P_{j}$. Since $P_{j}$ in $G_{i}$ is attached to $P_{j}$ in $G_{i+1}$, these $n+1$ copies of $P_{i}$ together form a path $P$ having $2 m(n+1)$ vertices. Since $|I \cap P|=m(n+1)$ it follows that $I \cap P$ must contain every second vertex of $P$, except possibly in one position where $I \cap P$ skips two vertices of $P$. There are only $n$ paths and $n+1$ copies of $G_{1}$, hence the pigeon-hole principle implies that in some copy $G_{y}$ of $G_{1}, I$ contains every second vertex on every path $P_{i}$. From now onward, we only consider such a copy $G_{y}$.

In $G_{y}$, for every $i \leq n, I$ contains every second vertex of $P_{i}$. We make an assignment to the variables of $\phi$ as follows. If $I$ contains all the odd numbered vertices of $P_{i}$, then $v_{i}$ is set to true; otherwise, $I$ contains all the even numbered vertices of $P_{i}$ and $v_{i}$ is set to false. We argue that this assignment satisfies $\phi$. Indeed, consider any clause $C_{j}$, and look at the gadget $\widehat{C}_{j}$. We know that $I$ contains $\left|C_{j}\right|+2$ vertices from $\widehat{C}_{j}$, and hence $I$ must contain a vertex $\ell_{a}$ in $\widehat{C}_{j}$ corresponding to a literal of $C_{j}$. Suppose $\ell_{a}$ is a literal of $v_{i}$. Since $I$ contains $\ell_{a}$, if $\ell_{a}$ occurs positively in $C_{j}$, then $I$ can not contain $p_{i}^{2 j}$, and hence $v_{i}$ is true. Similarly, if $\ell_{a}$ occurs negatively in $C_{j}$, then $I$ cannot contain $p_{i}^{2 j-1}$, and hence $v_{i}$ is false. In both cases, $v_{i}$ satisfies $C_{j}$, and hence all clauses of $\phi$ are satisfied by the assignment.

Lemma 3. $\mathbf{p w}(G) \leq n+4$.

Proof. We give a mixed search strategy to clean $G$ using $n+3$ searchers. For every $i$, we place a searcher on the first vertex of $P_{i}$ in $G_{1}$. The $n$ searchers slide along the paths $P_{1}, \ldots P_{n}$ in $m$ rounds. In round $j$ each searcher $i$ starts on $p_{i}^{2 j-1}$. Then, for every variable $v_{i}$ that occurs positively in $C_{j}$, the searcher $i$ slides forward to $p_{i}^{2 j}$. Observe that at this point there is a searcher on every neighbour of the gadget $\widehat{C}_{j}$. This gadget can now be cleaned with three additional searchers. After $\widehat{C}_{j}$ is clean, the additional three searchers are removed, and each of the $n$ searchers on the paths $P_{1}, \ldots P_{n}$ slides forward along these paths, such that searcher $i$ stands on $p_{i}^{2(j+1)}$. At that point, the next round commences. When the searchers have cleaned $G_{1}$ they slide onto the first vertex of $P_{1} \ldots P_{n}$ in $G_{2}$. Then, they proceed to clean $G_{2}, \ldots, G_{n+1}$ in the same way that $G_{1}$ was cleaned. Now applying Proposition 1, we get that $\mathbf{p w}(G) \leq n+4$.

The construction, together with Lemmata 1, 2 and 3 proves Theorem 1.

\section{DOMINATING SET}

A dominating set of a graph $G$ is a set $S \subseteq V(G)$ such that $V(G)=N[S]$. In the Dominating SET problem, we are given a graph $G$ and the objective is to find a dominating set of minimum size.

The basic idea for this reduction is similar to the one for InDEPENDENT SET. However, we need one more new idea here, which will also be used in other reductions. We group variables into an appropriate number of groups of size at most $\beta=\left\lfloor\log 3^{p}\right\rfloor$, where $p$ is a constant depending only on $\epsilon$. Then, for every group, we make a gadget such that an assignment on the group should correspond to a selection on the gadget. These group gadgets are then connected to clause gadgets so that every assignment on the group that satisfies the clause results in some desired outcome. 


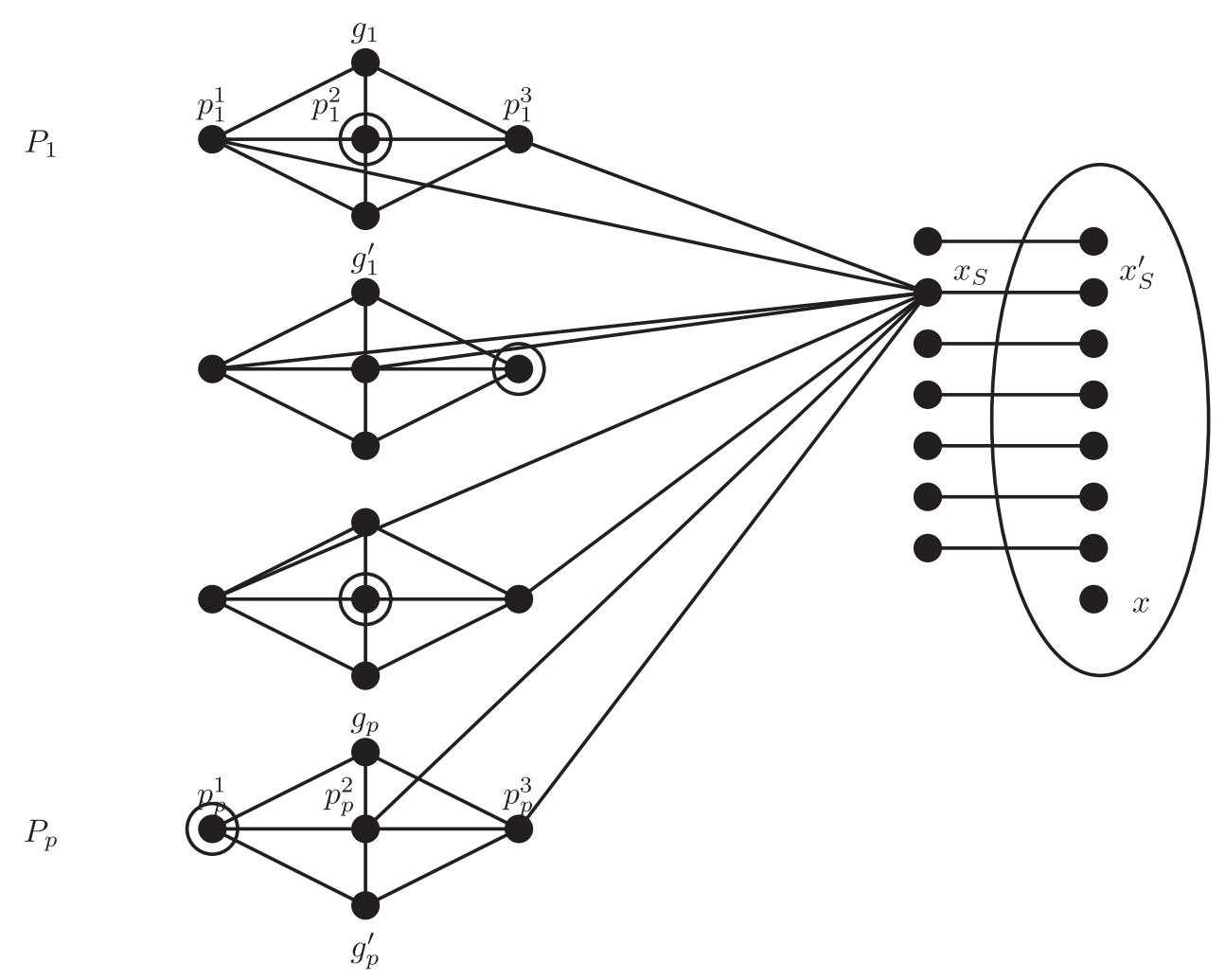

Fig. 2. Reduction to Dominating SET: group gadget $\widehat{B}$. The set $S$ is shown by the circled vertices.

Theorem 2. If Dominating Set can be solved in $O^{*}((3-\epsilon) \mathbf{p w}(G))$ time for some $\epsilon>0$, then SAT can be solved in $O^{*}\left((2-\delta)^{n}\right)$ time for some $\delta>0$.

Construction. Given $\epsilon<1$ and an instance $\phi$ to SAT, we construct a graph $G$ as follows. We first choose an integer $p$ depending only on $\epsilon$. Exactly how $p$ is chosen will be discussed in the proof of Theorem 2. We group the variables of $\phi$ into groups $F_{1}, F_{2}, \ldots, F_{t}$, each of size at most $\beta=\left\lfloor\log 3^{p}\right\rfloor$. Hence, $t=\lceil n / \beta\rceil$. We now proceed to describe a "group gadget" $\widehat{B}$, which is central in our construction.

To build the group gadget $\widehat{B}$, we introduce $p$ paths $P_{1}, \ldots, P_{p}$, where the path $P_{i}$ contains the vertices $p_{i}^{1}, p_{i}^{2}$, and $p_{i}^{3}$ (see Figure 2). To each path $P_{i}$, we attach two guards $g_{i}$ and $g_{i}^{\prime}$, both of which are neighbours to $p_{i}^{1}, p_{i}^{2}$, and $p_{i}^{3}$. When the gadgets are attached to each other, the guards will not have any neighbours outside of their own gadget $\widehat{B}$, and will ensure that at least one vertex out of $p_{i}^{1}, p_{i}^{2}$ and $p_{i}^{3}$ are chosen in any minimum size dominating set of $G$. Let $P$ be the vertex set containing all the vertices on the paths $P_{1}, \ldots, P_{p}$. For every subset $S$ of $P$ that picks exactly one vertex from each path $P_{i}$, we introduce two vertices $x_{S}$ and $x_{S}^{\prime}$, where $x_{S}$ is adjacent to all vertices of $P \backslash S$ (all those vertices that are on paths and not in $S$ ) and $x_{S}^{\prime}$ is only adjacent to $x_{S}$. We conclude the construction of $\widehat{B}$ by making all the vertices $x_{S}^{\prime}$ (for every set $S$ ) adjacent to each other, that is making them into a clique, and adding a guard $x$ adjacent to $x_{S}^{\prime}$ for every set $S$. In other words, the $x_{S}^{\prime}$ 's together with $x$ form a clique and all the neighbors of $x$ reside in this clique.

We construct the graph $G$ as follows (see Figure 3). For every group $F_{i}$ of variables, we introduce $m(2 p t+1)$ copies of the gadget $\widehat{B}$, call them $\widehat{B}_{i}^{j}$ for $1 \leq j \leq m(2 p t+1)$. We can imagine 


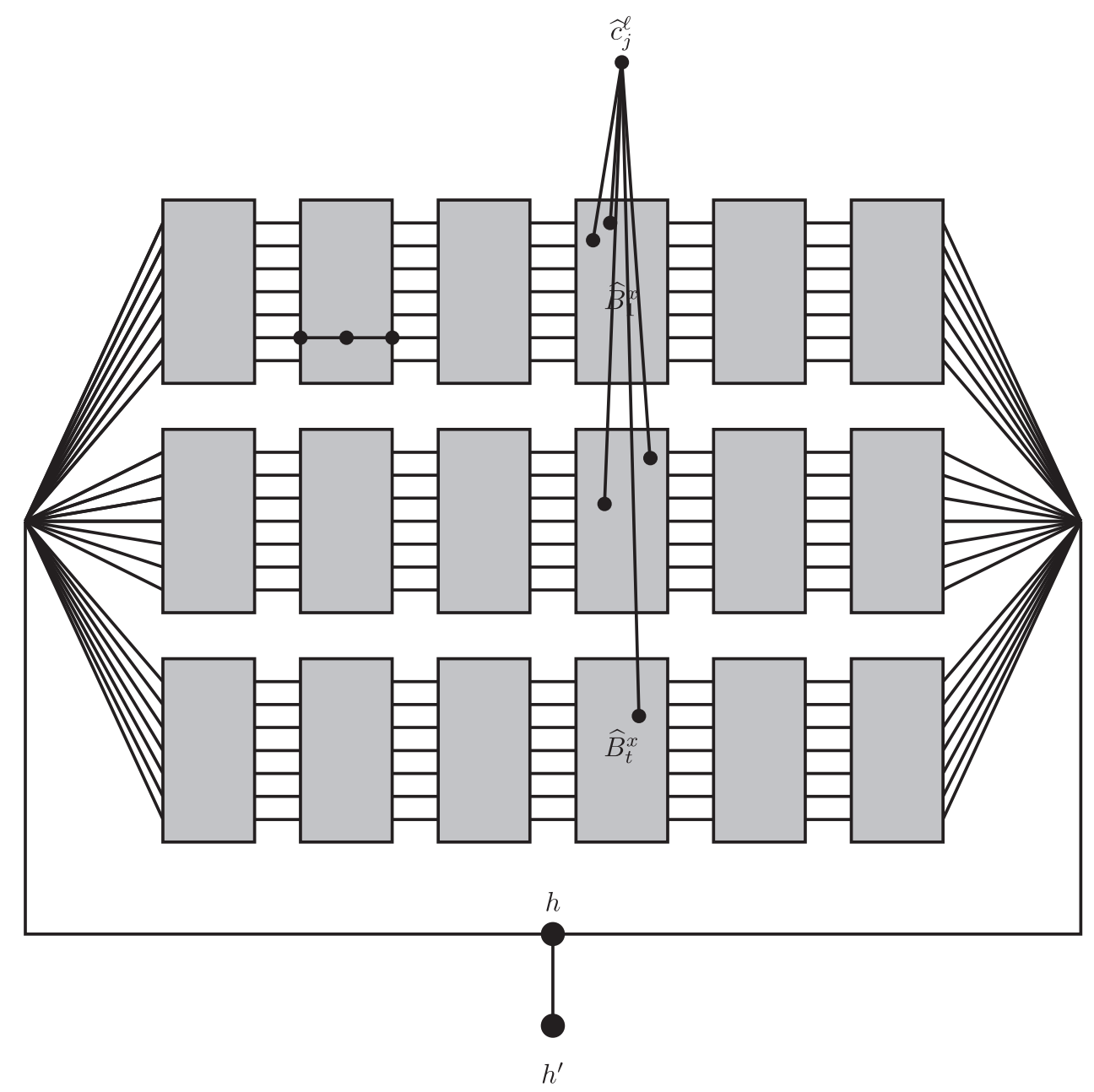

Fig. 3. Reduction to Dominating SET: arranging the group gadgets. Note that $x=m \ell+j$, thus $\widehat{c}_{j}^{\ell}$ is attached to vertices in $\widehat{B}_{1}^{x}, \ldots, \widehat{B}_{t}^{x}$.

these $t \cdot m(2 p t+1)$ gadgets arranged in $t$ rows and $m(2 p t+1)$ columns, with the columns being divided into $2 p t+1$ regions of $m$ columns each. For every fixed $i \leq t$, we connect the gadgets $\widehat{B}_{i}^{1}, \widehat{B}_{i}^{2} \ldots, \widehat{B}_{i}^{m(2 p t+1)}$ in a pathlike manner. In particular, for every $j<m(2 p t+1)$ and every $\ell \leq p$, we make an edge between $p_{\ell}^{3}$ in the gadget $\widehat{B}_{i}^{j}$ with $p_{\ell}^{1}$ in the gadget $\widehat{B}_{i}^{j+1}$. Now, we introduce two new vertices $h$ and $h^{\prime}$, with $h$ adjacent to $h^{\prime}, p_{j}^{1}$ in $\widehat{B}_{i}^{1}$ for every $i \leq t, j \leq p$ and to $p_{j}^{3}$ in $\widehat{B}_{i}^{m(2 p t+1)}$ for every $i \leq t, j \leq p$. That is, for all $1 \leq i \leq t, h$ is adjacent to the first and last vertices of "long paths" obtained after connecting the gadgets $\widehat{B}_{i}^{1}, \widehat{B}_{i}^{2} \ldots, \widehat{B}_{i}^{m(2 p t+1)}$ in a pathlike manner.

For every $1 \leq i \leq t$ and to every assignment of the variables in the group $F_{i}$, we designate a subset $S$ of $P$ in the gadget $\widehat{B}$ that picks exactly one vertex from each path $P_{j}$. Since there are at most $2^{\beta}$ different assignments to the variables in $F_{i}$, and there are $3^{p} \geq 2^{\beta}$ such sets $S$, we can assign a unique set to each assignment. Of course, the same set $S$ can correspond to one assignment of the group $F_{1}$ and some other assignment of the group $F_{2}$. Recall that the clauses of $\phi$ are $C_{1}, \ldots, C_{m}$. 
For every clause $C_{j}$, we introduce $2 p t+1$ vertices $\widehat{c}_{j}^{\ell}$, one for each $0 \leq \ell<2 p t+1$, corresponding to the $2 p t+1$ regions. The vertex $\widehat{c}_{j}^{\ell}$ will be connected to the gadgets $\widehat{B}_{i}^{m \ell+j}$ for every $1 \leq i \leq t$ (which appear in the $\ell$ th region). In particular, for every assignment of the variables in the group $F_{i}$ that satisfy the clause $C_{j}$, we consider the subset $S$ of $P$ that corresponds to the assignment. For every $0 \leq \ell<2 p t+1$, we make $x_{S}^{\prime}$ in $\widehat{B}_{i}^{m \ell+j}$ adjacent to $\widehat{c}_{j}^{\ell}$. The best way to view this is that every clause $C_{j}$ has $2 p t+1$ private gadgets in the $i$-row, $\widehat{B}_{i}^{j}, \widehat{B}_{i}^{m+j}, \ldots, \widehat{B}_{i}^{m 2 p t+j}$, one in each region. Now, we have $2 p t+1$ vertices corresponding to the clause $C_{j}$, each connected to one of these gadgets. This concludes the construction of $G$.

LEMma 4. If $\phi$ has a satisfying assignment, then $G$ has a dominating set of size $(p+1) t m(2 p t+$ 1) +1 .

Proof. Given a satisfying assignment to $\phi$, we construct a dominating set $D$ of $G$ that contains the vertex $h$ and exactly $p+1$ vertices in each gadget $\widehat{B}_{i}^{j}$. For each group $F_{i}$ of variables, we consider the set $S$ that corresponds to the restriction of the assignment to the variables in $F_{i}$. From each gadget $\widehat{B}_{i}^{j}$, we add the set $S$ to $D$ and also the vertex $x_{S}^{\prime}$ to $D$. It remains to argue that $D$ is indeed a dominating set. Clearly the size is bounded by $(p+1) \operatorname{tm}(2 p t+1)+1$, as the number of gadgets is $\operatorname{tm}(2 p t+1)$.

For a fixed $i \leq t$ and $j$ consider the vertices on the path $P_{j}$ in the gadgets $\widehat{B}_{i}^{\ell}$ for every $\ell \leq$ $m(2 p t+1)$. Together these vertices form a path of length $3 m(2 p t+1)$ and every third vertex of this path is in $S$. Thus, all vertices on this path are dominated by other vertices on the path, except perhaps for the first and last one. Both these vertices, however, are dominated by $h$.

Now, fix some $i \leq t$ and $\ell \leq m(2 p t+1)$ and consider the gadget $\widehat{B}_{i}^{\ell}$. Since $D$ contains some vertex on the path $P_{j}$, we have that for every $j$ both $g_{j}$ and $g_{j}^{\prime}$ are dominated. Furthermore, for every set $S^{*}$ not equal to $S$ that picks exactly one vertex from each $P_{j}$, vertex $x_{S^{*}}$ is dominated by some vertex on some $P_{j}$-namely by all vertices in $S \backslash S^{*} \neq \emptyset$. The last assertion follows, since $x_{S^{*}}$ is connected to all the vertices on the paths except $S^{*}$. On the other hand, $x_{S}$ is dominated by $x_{S}^{\prime}$, and $x_{S}^{\prime}$ also dominates all the other vertices $x_{S^{*}}^{\prime}$ for $S^{*} \neq S$, as well as the guard $x$.

The only vertices not yet accounted for are the vertices $\widehat{c}_{j}^{\ell}$ for every $j \leq m$ and $\ell<2 p t+1$. Fix a $j$ and a $\ell$ and consider the clause $C_{j}$. This clause contains a literal set to true, and this literal corresponds to a variable in the group $F_{i}$ for some $i \leq t$. Of course, the assignment to $F_{i}$ satisfies $C_{j}$. Let $S$ be the set corresponding to this assignment of $F_{i}$. By the construction of $D$, the dominating set contains $x_{S}^{\prime}$ in $\widehat{B}_{i}^{m \ell+j}$ and $x_{S}^{\prime}$ is adjacent to $\widehat{c}_{j}^{\ell}$. This concludes the proof.

LEMMA 5. If $G$ has a dominating set of size $(p+1) t m(2 p t+1)+1$, then $\phi$ has a satisfying assignment.

Proof. Let $D$ be a dominating set of $G$ of size at most $(p+1) t m(2 p t+1)+1$. Since $D$ must dominate $h^{\prime}$, without loss of generality, we can assume that $D$ contains $h$. Furthermore, inside every gadget $\widehat{B}_{i}^{\ell}, D$ must dominate all the guards, namely $g_{j}$ and $g_{j}^{\prime}$ for every $j \leq p$, and also $x$. Thus, $D$ contains at least $p+1$ vertices from each gadget $\widehat{B}_{i}^{\ell}$, which in turn implies that $D$ contains exactly $p+1$ vertices from each gadget $\widehat{B}_{i}^{\ell}$. The only way $D$ can dominate $g_{j}$ and $g_{j}^{\prime}$ for every $j$ and in addition dominate $x$ with only $p+1$ vertices if $D$ has one vertex from each $P_{j}, j \leq p$ and in addition contains some vertex in $N[x]$. Let $S$ be $D \cap P$ in $\widehat{B}_{i}^{\ell}$. Observe that $x_{S}$ is not dominated by $D \cap S$. The only vertex in $N[x]$ that dominates $x_{S}$ is $x_{S}^{\prime}$, and hence $D$ contains $x_{S}^{\prime}$.

Now, we want to show that for every $1 \leq i \leq t$ there exists one $0 \leq \ell \leq 2 t p$ such that for fixed $i, D \cap P$ is same in all the gadgets $\widehat{B}_{i}^{m \ell+r}$ for every $1 \leq r \leq m$, i.e., it is the same in every gadget of the $i$ th row in the $\ell$ th region. Consider a gadget $\widehat{B}_{i}^{\ell}$ and its follower, $\widehat{B}_{i}^{\ell+1}$. Let $S$ be $D \cap P$ in $\widehat{B}_{i}^{\ell}$ and 
$S^{\prime}$ be $D \cap P$ in $\widehat{B}_{i}^{\ell+1}$. Observe that if $S$ contains $p_{j}^{a}$ in $\widehat{B}_{i}^{\ell}$ and $p_{j}^{b}$ in $\widehat{B}_{i}^{\ell+1}$ then we must have $b \leq a$. We call a consecutive pair $b a d$ if for some $j \leq p, D$ contains $p_{j}^{a}$ in $\widehat{B}_{i}^{\ell}$ and $p_{j}^{b}$ in $\widehat{B}_{i}^{\ell+1}$ and $b<a$. Hence, for a fixed $i$, we can at most have $2 p$ consecutive bad pairs, spoiling at most $2 p$ regions. Now, we mark all the bad pairs that occur among the gadgets corresponding to some $F_{i}$. This way we can mark only $2 t p$ bad pairs. Thus, by the pigeon hole principle, there exists an $\ell \in\{0, \ldots, 2 t p\}$ such that there are no bad pairs in $\widehat{B}_{i}^{m \ell+r}$ for all $1 \leq i \leq t$ and $1 \leq r \leq m$.

We make an assignment $\phi$ by reading off $D \cap P$ in each gadget $\widehat{B}_{i}^{m \ell+1}$. In particular, for every group $F_{i}$, we consider $S=D \cap P$ in the gadget $\widehat{B}_{i}^{m \ell+1}$. This set $S$ corresponds to an assignment of $F_{i}$, and this is the assignment of $F_{i}$ that we use. It remains to argue that every clause $C_{r}$ is satisfied by this assignment.

Consider the vertex $\widehat{c}_{\ell}^{r}$. We know that it is dominated by some $x_{S}^{\prime}$ in a gadget $\widehat{B}_{i}^{m \ell+r}$. The set $S$ corresponds to an assignment of $F_{i}$ that satisfies the clause $C_{r}$. Because $D \cap P$ remains unchanged in all gadgets from $\widehat{B}_{i}^{m \ell+1}$ to $\widehat{B}_{i}^{m \ell+r}$, this is exactly the assignment $\phi$ restricted to the group $F_{i}$. This concludes the proof.

Lemma 6. $\mathbf{p w}(G) \leq t p+O\left(3^{p}\right)$.

Proof. We give a mixed search strategy to clean the graph with $t p+O\left(3^{p}\right)$ searchers. For a gadget $\widehat{B}$, we call the vertices $p_{j}^{1}$ and $p_{j}^{3}, 1 \leq j \leq p$, as entry vertices and exit vertices, respectively. We search the graph in $m(2 t p+1)$ rounds. In the beginning of round $\ell$ there are searchers on the entry vertices of the gadgets $\widehat{B}_{i}^{\ell}$ for every $i \leq t$. Let $1 \leq a \leq m$ and $0 \leq b<2 t p+1$ be integers such that $\ell=a+m b$. We place a searcher on $\vec{c}_{a}^{b}$. Then, for each $i$ between 1 and $p$ in turn, we first put searchers on all vertices of $\widehat{B}_{i}^{\ell}$ and then remove all the searchers from $\widehat{B}_{i}^{\ell}$ except for the ones standing on the exit vertices. After all gadgets $\widehat{B}_{1}^{\ell} \ldots \widehat{B}_{t}^{\ell}$ have been cleaned in this manner, we can remove the searcher from $\widehat{c}_{a}^{b}$. To commence the next round, the searchers slide from the exit positions of $\widehat{B}_{i}^{\ell}$ to the entry positions of $\widehat{B}_{i}^{\ell+1}$ for every $i$. In total, at most $t p+|V(\widehat{B})|+1 \leq$ $t p+O\left(3^{p}\right)$ searchers are used simultaneously. This together with Proposition 1 give the desired upperbound on the pathwidth.

Proof (of Theorem 2). Suppose Dominating Set can be solved in $O^{*}\left((3-\epsilon)^{\mathbf{p w}(G)}\right)=$ $O^{*}\left(3^{\lambda \mathbf{p w}(G)}\right)$ time, where $\lambda=\log _{3}(3-\epsilon)<1$. We choose $p$ large enough such that $\lambda \cdot \frac{p}{\lfloor p \log 3\rfloor}=$ $\frac{\delta^{\prime}}{\log 3}$ for some $\delta^{\prime}<1$. Given an instance of SAT, we construct an instance of Dominating SET using the above construction and the chosen value of $p$. Then, we solve the Dominating SET instance using the $O^{*}\left(3^{\lambda \mathbf{p w}(G)}\right)$ time algorithm. Correctness is ensured by Lemmata 4 and 5 . Lemma 6 yields that the total time taken is upper bounded by $O^{*}\left(3^{\lambda \mathbf{p w}(G)}\right) \leq O^{*}\left(3^{\lambda(t p+f(\lambda))}\right) \leq O^{*}\left(3^{\lambda \frac{n p}{[p \log 3]}}\right) \leq$ $O^{*}\left(3^{\delta^{\prime} \frac{n}{\log 3}}\right) \leq O^{*}\left(2^{\delta^{\prime \prime} n}\right)=O^{*}\left((2-\delta)^{n}\right)$, for some $\delta^{\prime \prime}, \delta<1$. This concludes the proof.

\section{MAX CUT}

A cut in a graph $G$ is a partition of $V(G)$ into $V_{0}$ and $V_{1}$. The cut-set of the cut is the set of edges whose one end point is in $V_{0}$ and the other in $V_{1}$. We say that an edge is crossing this cut if it has one endpoint in $V_{0}$ and one in $V_{1}$, that is, the edge is in the cut-set. The size of the cut is the number of edges in $G$ that are crossing this cut. If the edges of $G$ have positive integer weights, then the weight of the cut is the sum of the weights of edges that are crossing the cut. In the Max CuT problem, we are given a graph $G$ together with an integer $t$ and asked whether there is a cut of $G$ of size at least $t$. In the Weighted MAx Cut problem every edge has a positive integer weight and the objective is to find a cut of weight at least $t$. 
Theorem 3. If MAX Cut can be solved in $O^{*}\left((2-\epsilon)^{\mathbf{p w}(G)}\right)$ for some $\epsilon>0$, then SAT can be solved in $O^{*}\left((2-\delta)^{n}\right)$ time for some $\delta>0$.

Construction. Given an instance $\phi$ of SAT, we first construct an instance $G_{w}$ of WeIGHTED MAx CUt as follows. We later explain how to obtain an instance of unweighted MAX CUT from here.

We start with introducing a vertex $x_{0}$. Without loss of generality, we will assume that $x_{0} \in V_{0}$ in every solution. We introduce a vertex $\widehat{v}_{i}$ for each variable $v_{i}$. For every clause $C_{j}$, we create a gadget as follows. We introduce a path $\widehat{P}_{j}$ having $4\left|C_{j}\right|$ vertices. All the edges on $\widehat{P}_{j}$ have weight $3 n$. Now, we make the first and last vertex of $\widehat{P}_{j}$ adjacent to $x_{0}$ with an edge of weight $3 n$. Thus the path $\widehat{P}_{j}$ plus the edges from the first and last vertex of $\widehat{P}_{j}$ to $x_{0}$ form an odd cycle $\widehat{C}_{j}$. We will say that the first, third, fifth, and so on, vertices are on odd positions on $\widehat{P}_{j}$ while the remaining vertices are on even positions. For every variable $v_{i}$ that appears positively in $C_{j}$, we select a vertex $p$ at an even position (but not the last vertex) on $\widehat{P}_{j}$ and make $\widehat{v}_{i}$ adjacent to $p$ and $p$ 's successor on $\widehat{P}_{j}$ with edges of weight 1 . For every variable $v_{i}$ that appears negatively in $C_{j}$, we select a vertex $p$ at an odd position on $\widehat{P}_{j}$ and make $\widehat{v}_{i}$ adjacent to $p$ and $p$ 's successor on $\widehat{P}_{j}$ with edges of weight 1 . We make sure that each vertex on $\widehat{P}_{j}$ receives an edge at most once in this process. There are more than enough vertices on $\widehat{P}_{j}$ to accommodate all the edges incident to vertices corresponding to variables in the clause $C_{j}$. We create such a gadget for each clause and set $t=m+(12 n+1) \sum_{j=1}^{m}\left|C_{j}\right|$. This concludes the construction.

Lemma 7. If $\phi$ is satisfiable, then $G_{w}$ has a cut of weight at least $t$.

Proof. Suppose $\phi$ is satisfiable. We put $x_{0}$ in $V_{0}$ and for every variable $v_{i}$, we put $\widehat{v}_{i}$ in $V_{1}$ if $v_{i}$ is true and $\widehat{v}_{i}$ in $V_{0}$ if $v_{i}$ is false. For every clause $C_{j}$, we proceed as follows. Let us choose a true literal of $C_{j}$ and suppose that this literal corresponds to a vertex $p_{j}$ on $\widehat{P}_{j}$. We put the first vertex on $\widehat{P}_{j}$ in $V_{1}$, the second in $V_{0}$, and then we proceed along $\widehat{P}_{j}$ putting every second vertex into $V_{1}$ and $V_{0}$ until we reach $p_{j}$. The successor $p_{j}^{\prime}$ of $p_{j}$ on $\widehat{P}_{j}$ is put into the same set as $p_{j}$. Then, we continue along $\widehat{P}_{j}$ putting every second vertex in $V_{1}$ and $V_{0}$. Notice that even though $C_{j}$ may contain more than one literal that is set to true, we only select one vertex $p_{j}$ from the path $\widehat{P}_{j}$ and put $p_{j}$ and its successor on the same side of the partition. It remains to argue that this cut has weight at least $t$.

For every clause $C_{j}$ all edges on the path $\widehat{P}_{j}$ except for $p_{j} p_{j}^{\prime}$ are crossing, and the two edges to $x_{0}$ from the first and last vertex of $\widehat{P}_{j}$ are crossing as well. These edges contribute $12 n\left|C_{j}\right|$ to the weight of the cut. We know that $p_{j}$ corresponds to a literal that is set to true, and this literal corresponds to a variable $v_{i}$. If $v_{i}$ occurs positively in $C_{j}$, then $\widehat{v}_{i} \in V_{1}$ and $p_{j}$ is on an even position of $\widehat{P}_{j}$. Thus, both $p_{j}$ and its successor $p_{j}^{\prime}$ are in $V_{0}$, and hence both $\widehat{v}_{i} p_{j}$ and $\widehat{v}_{i} p_{j}^{\prime}$ are crossing, contributing 2 to the weight of the cut. For each of the remaining variables $v_{i^{\prime}}$ appearing in $C_{j}$, one of the two neighbours of $\widehat{v}_{i^{\prime}}$ on $\widehat{P}_{j}$ appear in $V_{0}$ and one in $V_{1}$, so exactly one edge from $v_{i^{\prime}}$ to $\widehat{P}_{j}$ is crossing. Thus the total weight of the cut is $t=\sum_{j=1}^{m}\left(12 n\left|C_{j}\right|+\left|C_{j}\right|+1\right)=m+(12 n+1) \sum_{j=1}^{m}\left|C_{j}\right|$. This completes the proof.

LEMMA 8. If $G_{w}$ has a cut of weight at least $t$, then $\phi$ is satisfiable.

Proof. Let $\left(V_{0}, V_{1}\right)$ be a cut of $G$ of maximum weight, hence the weight of this cut is at least $t$. Without loss of generality, let $x_{0} \in V_{0}$. For every clause $C_{j}$, at least one edge of the odd cycle $\widehat{C}_{j}$ is not crossing. If more than one edge of this cycle is not crossing, then the total weight of the cut edges incident to the path $\widehat{P}_{j}$ is at most $3 n\left(4\left|C_{j}\right|-1\right)+2 n<12 n\left|C_{j}\right|$. In this case, we could change the partition $\left(V_{0}, V_{1}\right)$ such that all edges of $\widehat{P}_{j}$ are crossing and the first vertex of $\widehat{P}_{j}$ is in $V_{1}$. Using 
the new partition the weight of the crossing edges in the cycle $\widehat{C}_{j}$ is at least $12 n\left|C_{j}\right|$ and the edges not incident to $\widehat{P}_{j}$ are unaffected by the changes. This contradicts that $\left(V_{0}, V_{1}\right)$ was a maximum weight cut. Thus, it follows that exactly one edge of $\widehat{C}_{j}$ is not crossing.

Given the cut $\left(V_{0}, V_{1}\right)$, we set each variable $v_{i}$ to true if $\widehat{v}_{i} \in V_{1}$ and $v_{i}$ to false, otherwise. Consider a clause $C_{j}$ and a variable $v_{i}$ that appears in $C_{j}$. Let $u v$ be the edge of $\widehat{C}_{j}$ that is not crossing. If there is a vertex $\widehat{v}_{i}$ adjacent to both $u$ and $v$, then it is possible that both $\widehat{v}_{i} u$ and $\widehat{v}_{i} v$ are crossing. For every other variable $v_{i^{\prime}}$ in $C_{j}$, at most one of the edges from $\widehat{v}_{i^{\prime}}$ to $\widehat{P}_{j}$ is crossing. Thus, the weight of the edges that are crossing in the gadget $\widehat{C}_{j}$ is at most $(12 n+1)\left|C_{j}\right|+1$. Hence, to find a cut-set of weight at least $t$ in $G$, we need to have crossing edges in $\widehat{C}_{j}$ with sum of their weights exactly equal to $12 n\left|C_{j}\right|+\left|C_{j}\right|+1$. It follows that there is a vertex $\widehat{v}_{i}$ adjacent to both $u$ and $v$ such that both $\widehat{v}_{i} u$ and $\widehat{v}_{i} v$ are crossing.

If $v_{i}$ occurs in $C_{j}$ positively, then $u$ is on an even position, and hence $u \in V_{0}$. Since $\widehat{v}_{i} u$ is crossing it follows that $v_{i}$ is true and $C_{j}$ is satisfied. On the other hand, if $v_{i}$ occurs in $C_{j}$ negated then $u$ is on an odd position, and hence $u \in V_{1}$. Since $\widehat{v}_{i} u$ is crossing, it follows that $v_{i}$ is false and $C_{j}$ is satisfied. As this holds for each clause individually, this concludes the proof.

For every edge $e \in E\left(G_{w}\right)$, let $w_{e}$ be the weight of $e$ in $G_{w}$. We construct an unweighted graph $G$ from $G_{w}$ by replacing every edge $e=u v$ by $w_{e}$ paths from $u$ to $v$ on three edges. Let $W$ be the sum of the edge weights of all edges in $G_{w}$.

LEMma 9. G has a cut of size $2 W+t$ if and only if $G_{w}$ has a cut of weight at least $t$.

Proof. Given a partition of $V\left(G_{w}\right)$, we partition $V(G)$ as follows. The vertices of $G$ that also are vertices of $V(G)$ are partitioned in the same way as in $V\left(G_{w}\right)$. On each path of length 3 , if the endpoints of the path are in different sets, we can partition the middle vertices of the path such that all edges are cut. If the endpoints are in the same set, then we can only partition the middle vertices such that 2 out of the 3 edges are cut. The reverse direction is similar.

LEMMA 10. $\mathbf{p w}(G) \leq n+5$.

Proof. We give a search strategy to clean $G$ with $n+5$ searchers. We place one searcher on each vertex $\widehat{v}_{i}$ and one searcher on $x_{0}$. Then one can search the gadgets $\widehat{C}_{j}$ one by one. In $G_{w}$ it is sufficient to use 2 searchers for each $\widehat{C}_{j}$, whereas in $G$ after the edges have been replaced by multiple paths on three edges, we need 4 searchers. This combined with Proposition 1 gives the desired upper bound on the pathwidth of the graph.

The construction, together with Lemmata 7, 8, 9, and 10 proves Theorem 3.

\section{GRAPH COLORING}

A $q$-coloring of $G$ is a function $\mu: V(G) \rightarrow[q]$. A $q$-coloring $\mu$ of $G$ is proper if for every edge $u v \in E(G)$ we have $\mu(u) \neq \mu(v)$. In the $q$-Coloring problem, we are given as input a graph $G$ and the objective is to decide whether $G$ has a proper $q$-coloring. In the List ColORING problem, every vertex $v$ is given a list $L(v) \subseteq[q]$ of admissible colors. A proper list coloring of $G$ is a function $\mu: V(G) \rightarrow[q]$ such that $\mu$ is a proper coloring of $G$ that satisfies $\mu(v) \in L(v)$ for every $v \in V(G)$. In the $q$-List Coloring problem, we are given a graph $G$ together with a list $L(v) \subseteq[q]$ for every vertex $v$. The task is to determine whether there exists a proper list coloring of $G$.

A feedback vertex set of a graph $G$ is a set $S \subseteq V(G)$ such that $G \backslash S$ is a forest; we denote by $\mathbf{f v s}(G)$ the size of the smallest such set. It is well-known that $\mathbf{t w}(G) \leq \mathbf{f v s}(G)+1$. Unlike the other sections, where we give lower bounds for algorithms parameterized by $\mathbf{p w}(G)$, the following theorem gives also a lower bound for algorithms parameterized by $\mathbf{f v s}(G)$. Such a lower bound 
follows very naturally from the construction we are doing here, but not from the constructions in the other sections. It would be interesting to explore whether it is possible to prove tight bounds parameterized by $\mathbf{f v s}(G)$ for the problems considered in the other sections.

THEORem 4. Let $q$ be a fixed positive integer. If q-Coloring can be solved in $O^{*}\left((q-\epsilon)^{\mathbf{f v s}(G)}\right)$ or $O^{*}\left((q-\epsilon)^{\mathbf{p w}(G)}\right)$ time for some $\epsilon>0$, then SAT can be solved in $O^{*}\left((2-\delta)^{n}\right)$ time for some $\delta>0$.

Construction. We will show the result for List Coloring first, and then give a simple reduction that demonstrates that $q$-Coloring can be solved in $O^{*}\left((q-\epsilon)^{\mathbf{f v s}(G)}\right)$ time if and only if $q$-LisT COLORING can.

Depending on $\epsilon$ and $q$, we choose a parameter $p$. Now, given an instance $\phi$ to SAT, we will construct a graph $G$ with a list $L(v)$ for every $v$, such that $G$ has a proper list-coloring if and only if $\phi$ is satisfiable. Throughout the construction, we will call color 1-red, color 2-white, and color 3-black.

We start by grouping the variables of $\phi$ into $t$ groups $F_{1}, \ldots, F_{t}$ of size at most $\left\lfloor\log q^{p}\right\rfloor$. Thus $t=\left\lceil\frac{n}{\left\lfloor\log q^{p}\right\rfloor}\right\rceil$. We will call an assignment of truth values to the variables in a group $F_{i}$ a group assignment. We will say that a group assignment satisfies a clause $C_{j}$ of $\phi$ if $C_{j}$ contains at least one literal that is set to true by the group assignment. Notice that $C_{j}$ can be satisfied by a group assignment of a group $F_{i}$, even though $C_{j}$ also contains variables that are not in $F_{i}$.

For each group $F_{i}$, we introduce a set $V_{i}$ of $p$ vertices $v_{i}^{1}, \ldots, v_{i}^{p}$. The vertices in $V_{i}$ get full lists, that is, they can be colored by any color in [q]. The coloring of the vertices in $V_{i}$ will encode the group assignment of $F_{i}$. There are $q^{p} \geq 2^{\left|F_{i}\right|}$ possible colorings of $V_{i}$. Thus, to each possible group assignment of $F_{i}$, we attach a unique coloring of $V_{i}$. Notice that some colorings of $V_{i}$ may not correspond to any group assignments of $F_{i}$.

For each clause $C_{j}$ of $\phi$, we introduce a gadget $\widehat{C}_{j}$. The main part of $\widehat{C}_{j}$ is a long path $\widehat{P}_{j}$ that has one vertex for each group assignment that satisfies $\widehat{C}_{j}$. Notice that there are at most $t q^{p}$ possible group assignments, and that $q$ and $p$ are constants independent of the input $\phi$. The list of every vertex on $\widehat{P}_{j}$ is $\{$ red, white, black $\}$. We attach two vertices $p_{j}^{\text {start }}$ and $p_{j}^{\text {end }}$ to the start and end of $\widehat{P}_{j}$ respectively, and the two vertices are not counted as vertices of the path $\widehat{P}_{j}$ itself. The list of $p_{j}^{\text {start }}$ is $\{$ white $\}$. If $\left|V\left(\widehat{P}_{j}\right)\right|$ is even, then the list of $p_{j}^{e n d}$ is $\{$ white $\}$, whereas if $\left|V\left(\widehat{P}_{j}\right)\right|$ is odd then the list of $p_{j}^{e n d}$ is $\left\{\right.$ black\}. The intention is that to properly color $\widehat{P}_{j}$, one needs to use the color red at least once, and that once is sufficient. The position of the red-colored vertex on the path $\widehat{P}_{j}$ encodes how the clause $C_{j}$ is satisfied.

For every vertex $v$ on $\widehat{P}_{j}$, we proceed as follows. The vertex $v$ corresponds to some group assignment to $F_{i}$ that satisfies the clause $C_{j}$. This assignment in turn corresponds to a coloring of the vertices of $V_{i}$. Let this coloring be $\mu_{i}$. We build a connector whose role is to enforce that $v$ can be red only if coloring $\mu_{i}$ appears on $V_{i}$. To build the connector, for each vertex $v_{i}^{\ell} \in V_{i}$ and color $x \in[q] \backslash\left\{\mu_{i}\left(v_{i}^{\ell}\right)\right\}$, we do the following to enforce that if $v$ is red, then $v_{i}^{\ell}$ cannot have color $x$ (see Figure 5).

- If $x$ is red, then we introduce one vertex $w_{y}$ for every color $y$ except for red. We make $w_{y}$ adjacent to $v_{i}^{\ell}$ and the list of $w_{y}$ is $\{\mathrm{red}, y\}$. Then, we introduce a vertex $w$ that is adjacent to $v$ and to all vertices $w_{y}$. The list of $w$ is all of $[q]$.

- If $x$ is not red, then we introduce two vertices $w_{y}$ and $w_{y}^{\prime}$ for each color $y$ except for red. We make $w_{y}$ adjacent to $v_{i}^{\ell}$ and $w_{y}^{\prime}$ adjacent to $w_{y}$. The list of $w_{y}$ is $\{x$, red $\}$, while the list of $w_{y}^{\prime}$ is $\{y$, red $\}$. Finally, we introduce a vertex $w$ adjacent to $v$ and to $w_{y}^{\prime}$ for all $y$. The list of $w$ is all of $[q]$. 


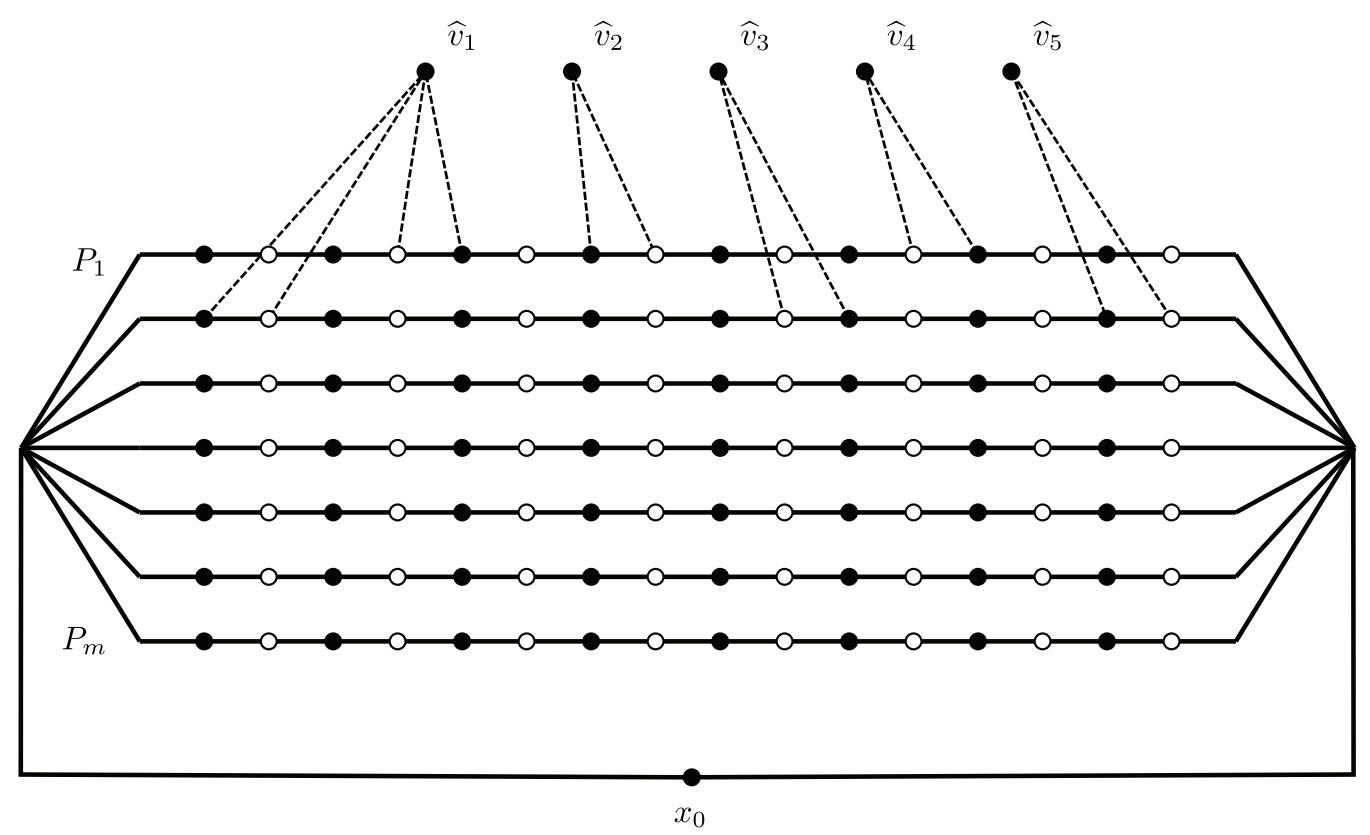

Fig. 4. Reduction to MAX Cut. The dashed edges have weight 1 and all the other edges have weight $3 n$. The odd and even positions of the paths $P_{j}$ are shown by black and white, respectively. As an example, we show potential connections corresponding to the clauses $C_{1}=\left(v_{1} \vee \bar{v}_{2} \vee v_{4}\right)$ and $C_{2}=\left(\bar{v}_{1} \vee v_{3} \vee \bar{v}_{5}\right)$.
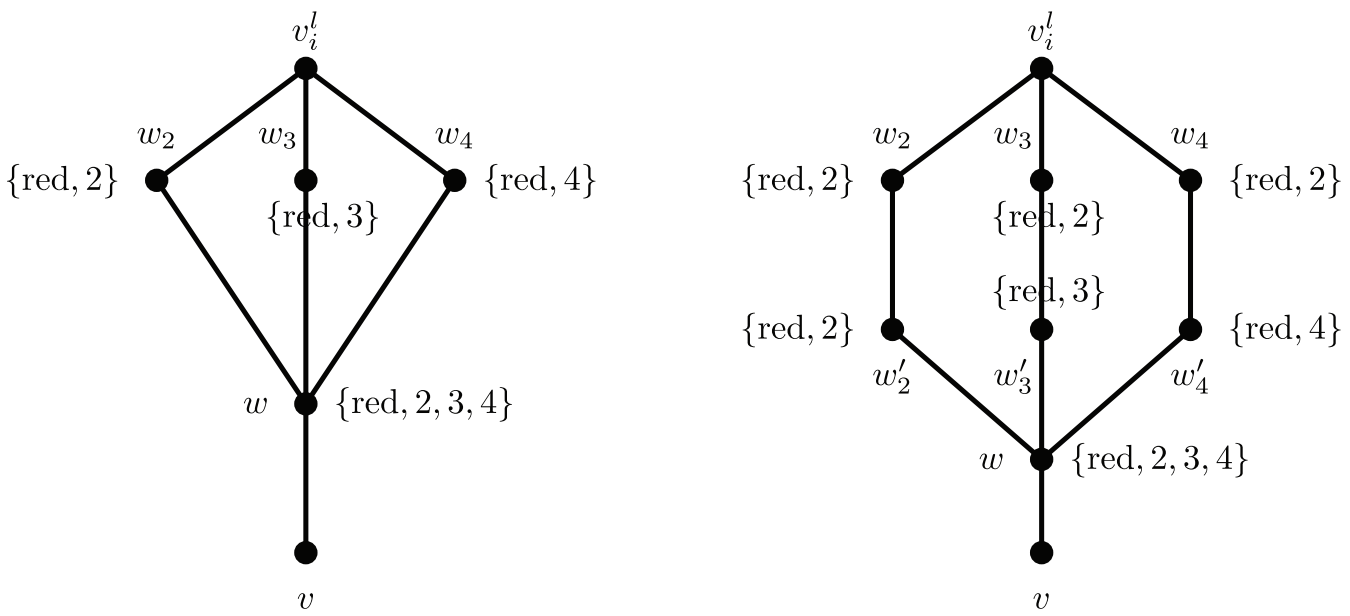

Fig. 5. Reduction to $q$-Coloring: the way the connector connects a vertex $v_{i}^{\ell}$ with $v$ for a particular "bad color" $x \in[q] \backslash\left\{\mu_{i}\left(v_{i}^{\ell}\right)\right\}$. The left side shows the case $x=$ red $=1$, the right side $x=2(q=4)$.

Notice that in the above construction we have reused the names $w, w_{y}$ and $w_{y}^{\prime}$ for many different vertices: in each connector, there is a separate vertex $w$ for each vertex $v_{i}^{\ell} \in V_{i}$ and color $x \in[q] \backslash\left\{\mu_{i}\left(v_{i}^{\ell}\right)\right\}$. Building a connector for each vertex $v$ on $\widehat{P}_{j}$ concludes the construction of the clause gadget $\widehat{C}_{j}$, and creating one such gadget for each clause concludes the construction of $G$ (see Figure 6). The following lemma summarizes the most important properties of the connector: 


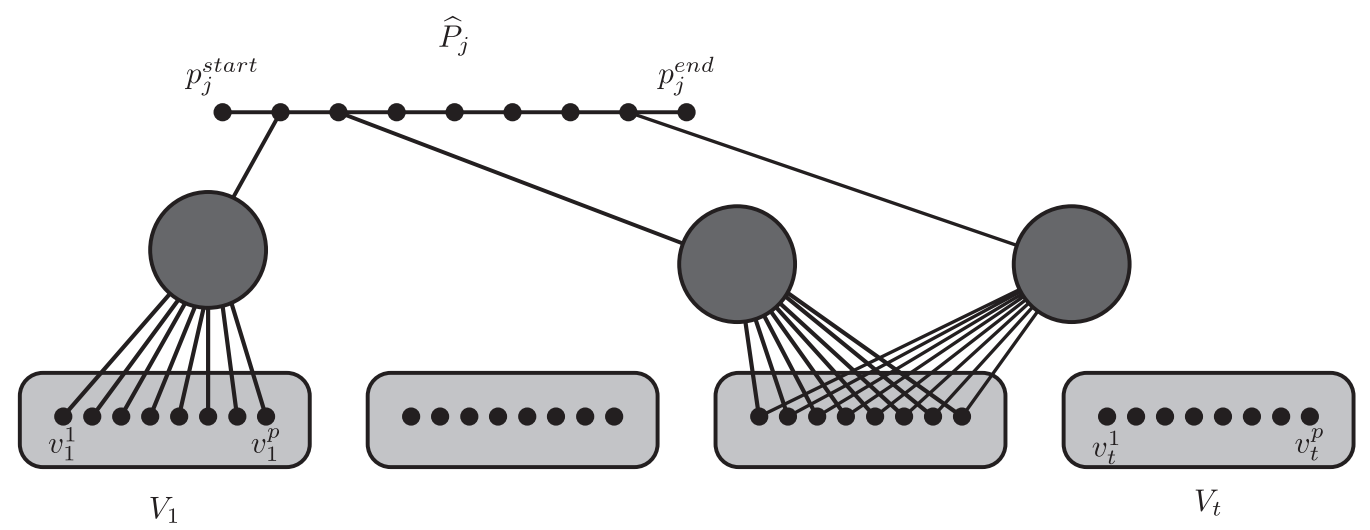

Fig. 6. Reduction to $q$-Coloring. The $t$ groups of vertices $V_{1}, \ldots, V_{t}$ represent the $t$ groups of variables $F_{1}$, $\ldots, F_{t}$ (each of size $\left\lceil\log q^{p}\right\rceil$ ). Each vertex of the clause path $\widehat{P}_{j}$ is connected to one group $V_{i}$ via a connector (multiple vertices on the path can be connected to the same group).

LEMMA 11. Consider the connector corresponding to a vertex $v$ on $\widehat{P}_{j}$ and a coloring $\mu_{i}$ of $V_{i}$.

(1) Any coloring on $V_{i}$ and any color $c \in\{$ white, black $\}$ on $v$ can be extended to the rest of the connector.

(2) Coloring $\mu_{i}$ on $V_{i}$ and any color $c \in\{$ red, white, black $\}$ on $v$ can be extended to the rest of the connector.

(3) In any coloring of the connector, if $v$ is red, then $\mu_{i}$ appears on $V_{i}$.

Proof. 1. For each vertex $v_{i}^{\ell} \in V_{i}$ and color $x \in[q] \backslash\left\{\mu_{i}\left(v_{i}^{\ell}\right)\right\}$, we do the following:

- If $x$ is red, then in the construction of $\widehat{C}_{j}$ we introduced a vertex $w_{y}$ with list $\{y$, red $\}$ for every color $y \neq$ red adjacent to $v_{i}^{\ell}$, and a vertex $w$ with list $[q]$ adjacent to $w_{y}$ for every $y \neq$ red. If $v_{i}^{\ell}$ is colored red, then we color each vertex $w_{y}$ with $y$ and $w$ with red. Notice that $w$ is adjacent to $v$, but $v$ is colored either white or black, so it is safe to color $w$ red. If, on the other hand, $v_{i}^{\ell}$ is not colored red, then we can color $w_{y}$ red for every $y$. Then, all the neighbours of $w$ have been colored with red, except for $v$, which has been colored white or black. Thus, it is safe to color $w$ with the color out of black and white that was not used to color $v$.

- If $x$ is not red, then in the construction of $\widehat{C}_{j}$, we introduced two vertices $w_{y}$ and $w_{y}^{\prime}$ for each color $y$ except for red, and also introduced a vertex $w$. The vertices $w_{y}$ are adjacent to $v_{i}^{\ell}$ and for every $y \neq$ red, the vertex $w_{y}^{\prime}$ is adjacent to $w_{y}$. Finally, $w$ is adjacent to all the vertices $w_{y}^{\prime}$ and to $v$. For every $y$, the list of $w_{y}$ is $\{x$, red $\}$ while the list of $w_{y}^{\prime}$ is $\{y$, red $\}$. The list of $w$ is $[q]$. If $v_{i}^{\ell}$ is colored with $x$, then we let $w_{y}$ take color red and $w_{y}^{\prime}$ take color $y$ for every $y \neq$ red. We color $w$ with red. In the case that $v_{i}^{\ell}$ is colored with a color different from $x$, we let $w_{y}$ be colored with $x$ and $w_{y}^{\prime}$ be colored red for every $y \neq$ red. Finally, all the neighours of $w$ except for $v$ have been colored red, while $v$ is colored with either black or white. According to the color of $v$, we can either color $w$ black or white.

2. We can assume that $v$ is red, otherwise, we are done by the previous statement. For each vertex $v_{i}^{\ell} \in V_{i}$ and color $x \in[q] \backslash\left\{\mu_{i}\left(v_{i}^{\ell}\right)\right\}$, we do the following. 
- If $x$ is red, then in the construction of $\widehat{C}_{j}$ we introduced a vertex $w_{y}$ with list $\{y$, red $\}$ for every color $y \neq$ red adjacent to $v_{i}^{\ell}$, and a vertex $w$ with list $[q]$ adjacent to $w_{y}$ for every $y \neq$ red. Since $v_{i^{\prime}}^{\ell}$ is not colored red by $\mu_{i}$, we can color $w_{y}$ red for every $y$. Then, all the neighbours of $w$ including $v$ have been colored with red and it is safe to color $w$ with white.

- If $x$ is not red, then in the construction of $\widehat{C}_{j}$, we introduced two vertices $w_{y}$ and $w_{y}^{\prime}$ for each color $y$ except for red, and also introduced a vertex $w$. The vertices $w_{y}$ are adjacent to $v_{i}^{\ell}$ and for every $y \neq$ red the vertex $w_{y}^{\prime}$ is adjacent to $w_{y}$. Finally, $w$ is adjacent to all the vertices $w_{y}^{\prime}$ and to $v$. For every $y$, the list of $w_{y}$ is $\{x$, red $\}$ while the list of $w_{y}^{\prime}$ is $\{y$, red $\}$. The list of $w$ is $[q]$. Since $\mu_{i}$ colors $v_{i}^{\ell}$ with a color different from $x$, we let $w_{y}$ be colored with $x$ and $w_{y}^{\prime}$ be colored red for every $y \neq$ red. Finally, all the neighours of $w$, including $v$, have been colored red so it is safe to color $w$ white.

3. Suppose for contradiction that $v$ is red, but some vertex $v_{i}^{\ell} \in V_{i}$ has been colored with a color $x \neq \mu_{i}\left(v_{i}^{\ell}\right)$. There are two cases. If $x$ is red, then in the construction, we introduced vertices $w_{y}$ adjacent to $v_{i}^{\ell}$ for every color $y \neq$ red. Also, we introduced a vertex $w$ adjacent to $v$ and to $w_{y}$ for each $y \neq$ red. The list of $w_{y}$ is $\{$ red, $y\}$, and hence $w_{y}$ must have been colored $y$ for every $y \neq$ red. But then $w$ is adjacent to $v$, which is colored red, and to $w_{y}$, which is colored $y$ for every $y \neq$ red. Thus, vertex $w$ has all colors in its neighborhood, a contradiction. In the case when $x$ is not red, then in the construction, we introduced two vertices $w_{y}$ and $w_{y}^{\prime}$ for each $y \neq$ red. Each $w_{y}$ was adjacent to $v_{i}^{\ell}$ and had $\{x$, red $\}$ as its list. Since $v_{i}^{\ell}$ is colored $x$, all the $w_{y}$ vertices must be colored red. For every $y \neq$ red, we have that $w_{y}^{\prime}$ is adjacent to $w_{y}$ and has $\{$ red, $y\}$ as its list. Hence, for every $y \neq$ red, the vertex $w_{y}^{\prime}$ is colored with $y$. But, in the construction, we also introduced a vertex $w$ adjacent to $v$ and to $w_{y}^{\prime}$ for each $y \neq$ red. Thus again, vertex $w$ has all colors in its neighbourhood, a contradiction.

\section{LEMma 12. If $\phi$ is satisfiable, then $G$ has a proper list-coloring.}

Proof. Starting from a satisfying assignment of $\phi$, we construct a coloring $\gamma$ of $G$. The assignment to $\phi$ corresponds to a group assignment to each group $F_{i}$. Each group assignment corresponds to a coloring of $V_{i}$. For every $i$, we let $\gamma$ color the vertices of $V_{i}$ using the coloring corresponding to the group assignment of $F_{i}$.

Now, we show how to complete this coloring to a proper coloring of $G$. Since the gadgets $\widehat{C}_{j}$ are pairwise disjoint, and there are no edges going between them, it is sufficient to show that we can complete the coloring for every gadget $\widehat{C}_{j}$. Consider the clause $C_{j}$. The clause contains a literal that is set to true, and this literal belongs to a variable in some group $F_{i}$. The group assignment of $F_{i}$ satisfies the clause $C_{j}$. Thus, there is a vertex $v$ on $\widehat{P}_{j}$ that corresponds to this assignment. We set $\gamma(v)$ as red (that is, $\gamma$ colors $v$ red), $p_{j}^{\text {start }}$ is colored white and $p_{j}^{e n d}$ is colored with its only admissible color, namely black if $\left|V\left(\widehat{P}_{j}\right)\right|$ is even and white if $\left|V\left(\widehat{P}_{j}\right)\right|$ is odd. The remaining vertices of $\widehat{P}_{j}$ are colored alternatingly white or black. By Lemma 11(2), the coloring can be extended to every vertex of the connector between $V_{i}$ and $v$ : the coloring appearing on $V_{i}$ is the coloring $\mu_{i}$ corresponding to the group assignment $F_{i}$. For every other vertex $u$ on $\widehat{P}_{j}$, the color of $u$ is black or white, thus Lemma 11(1) ensures that the coloring can be extended to any connector on $u$.

As this procedure can be repeated to color the gadget $\widehat{C}_{j}$ for every clause $C_{j}$, we can complete $\gamma$ to a proper list-coloring of $G$.

LEMmA 13. If $G$ has a proper list-coloring $\gamma$, then $\phi$ is satisfiable.

Proof. Given $\gamma$, we construct an assignment to the variables of $\phi$ as follows. For every group $F_{i}$ of variables, if $\gamma$ colors $V_{i}$ with a coloring that corresponds to a group assignment of $F_{i}$, then 
we set this assignment for the variables in $F_{i}$. Otherwise, we set all the variables in $F_{i}$ to false. We need to argue that this assignment satisfies all the clauses of $\phi$.

Consider a clause $C_{j}$ and the corresponding gadget $\widehat{C}_{j}$. By a simple parity argument, $\widehat{P}_{j}$ cannot be colored using only the colors black and white. Thus, some vertex $v$ on $\widehat{P}_{j}$ is colored red. The vertex $v$ corresponds to a group assignment of some group $F_{i}$ that satisfies $\widehat{C}_{j}$. As $v$ is red, Lemma 11(3) implies that $V_{i}$ is colored with the coloring $\mu_{i}$ that corresponds to this assignment. The construction then implies that our chosen assignment satisfies $C_{j}$. As this is true for every clause, this concludes the proof.

ObServation 1. The vertices $\bigcup_{i \leq t} V_{i}$ form a feedback vertex set of $G$. Furthermore, $\mathbf{p w}(G) \leq p t+4$

Proof. Observe that after removing $\bigcup_{i \leq t} V_{i}$, all that is left are the gadgets $\widehat{C}_{j}$, which do not have any edges between each other. Each such gadget is a tree, and hence $\bigcup_{i \leq t} V_{i}$ form a feedback vertex set of $G$. If we place a searcher on each vertex of $\bigcup_{i \leq t} V_{i}$, then it is easy to see that each gadget $\widehat{C}_{j}$ can be searched with 4 searchers. The pathwidth bound on $G$ follows using Proposition 1.

Lemma 14. If $q$-List Coloring can be solved in $O^{*}\left((q-\epsilon)^{\mathbf{f v s}(G)}\right)$ or $O^{*}\left((q-\epsilon)^{\mathbf{p w}(G)}\right)$ time for some $\epsilon>0$, then SAT can be solved in $O^{*}\left((2-\delta)^{n}\right)$ time for some $\delta>0$.

Proof. Let $O^{*}\left((q-\epsilon)^{\mathbf{f v s}(G)}\right)=O^{*}\left(q^{\lambda \mathbf{f v s}(G)}\right)$ time, where $\lambda=\log _{q}(q-\epsilon)<1$. We choose a sufficiently large $p$ such that $\delta^{\prime}=\lambda \frac{p}{p-1}<1$. Given an instance $\phi$ of SAT, we construct a graph $G$ using the construction above, and run the assumed $q$-List ColORING. Correctness follows from Lemmata 12 and 13. By Observation 1, the graph $G$ has a feedback vertex set of size $p\left\lceil\frac{n}{\lfloor p \log q\rfloor}\right\rceil$. The choice of $p$ implies that

$$
\lambda p\left\lceil\frac{n}{\lfloor p \log q\rfloor}\right\rceil \leq \lambda p \frac{n}{(p-1) \log q}+p \leq \delta^{\prime} \frac{n}{\log q}+p \leq \delta^{\prime \prime} n,
$$

for some $\delta^{\prime \prime}<1$. Hence, SAT can be solved in time $O^{*}\left(2^{\delta^{\prime \prime} n}\right)=O^{*}\left((2-\delta)^{n}\right)$, for some $\delta>0$. By Observation 1, we also know that $\mathbf{p w}(G) \leq p t+4$. Thus, the feedback vertex set size and the pathwidth of the constructed graph just differs by 4 . This implies that $q$-List Coloring cannot be solved in $O^{*}\left((q-\epsilon)^{\mathbf{p w}(G)}\right)$ time.

Finally, observe that we can reduce $q$-List-Coloring to $q$-Coloring by adding a clique $Q=$ $\left\{q_{1}, \ldots, q_{c}\right\}$ on $q$ vertices to $G$ and making $q_{i}$ adjacent to $v$ when $i \notin L(v)$. Any coloring of $Q$ must use $q$ different colors, and without loss of generality $q_{i}$ is colored with color $i$. Then, one can complete the coloring if and only if one can properly color $G$ using a color from $L(v)$ for each $v$. We can add the clique $Q$ to the feedback vertex set-this increases the size of the minimum feedback vertex set by $q$. Since $q$ is a constant independent of the input, this yields Theorem 4 .

\section{ODD CYCLE TRANSVERSAL}

An equivalent formulation of the MAX CUT problem is to ask for a bipartite subgraph with the maximum number of edges, which is the same as asking for a set of edges of minimum size whose deletion makes the graph bipartite. We can also consider the vertex-deletion version of this problem. An odd cycle transversal of a graph $G$ is a subset $S \subseteq V(G)$ such that $G \backslash S$ is bipartite. In the Odd Cycle Transversal problem, we are given a graph $G$ together with an integer $k$ and asked whether $G$ has an odd cycle transversal of size $k$.

Theorem 5. If Odd Cycle Transversal can be solved in $O^{*}((3-\epsilon) \mathbf{p w}(G))$ time for $\epsilon>0$, then SAT can be solved in $O^{*}\left((2-\delta)^{n}\right)$ time for some $\delta>0$. 


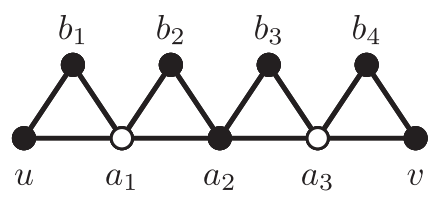

$A(u, v)$

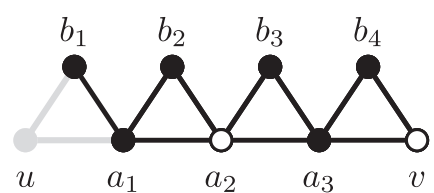

$A(u, v) \backslash\{u\}$

Fig. 7. Reduction to Odd Cycle Transversal. The arrow $A(u, v)$ from $u$ to $v$ with the passive odd cycle transversal shown in white (left) and the active odd cycle transversal of $A(u, v) \backslash\{u\}$ (right).

Construction. Given $\epsilon>0$ and an instance $\phi$ of SAT, we construct a graph $G$ as follows. We choose an integer $p$ based just on $\epsilon$. Exactly how $p$ is chosen will be discussed at the end of this section. We start by grouping the variables of $\phi$ into $t$ groups $F_{1}, \ldots, F_{t}$ of size at most $h=\left\lfloor\log 3^{p}\right\rfloor$. Thus, $t=\left\lceil\frac{n}{\left\lfloor\log 3^{p}\right\rceil}\right\rceil$. We will call an assignment of truth values to the variables in a group $F_{i}$ a group assignment. We will say that a group assignment satisfies a clause $C_{j}$ of $\phi$ if $C_{j}$ contains at least one literal that is set to true by the group assignment. Notice that $C_{j}$ can be satisfied by a group assignment of a group $F_{i}$ even though $C_{j}$ also contains variables that are not in $F_{i}$.

Now, we describe an auxiliary gadget that will be very useful in our construction (see Figure 7). For two vertices $u$ and $v$ by adding an arrow from $u$ to $v$, we will mean adding a path $u a_{1} a_{2} a_{3} v$ on four edges starting in $u$ and ending in $v$. Furthermore, we add four vertices $b_{1}, b_{2}, b_{3}$, and $b_{4}$ and edges $u b_{1}, b_{1} a_{1}, a_{1} b_{2}, b_{2} a_{2}, a_{2} b_{3}, b_{3} a_{3}, a_{3} b_{4}, b_{4} v$, and $b_{4} v$. Denote the resulting graph $A(u, v)$. None of the vertices in $A(u, v)$ except for $u$ and $v$ will receive any further neighbours throughout the construction of $G$. The graph $A(u, v)$ has the following properties, which are useful for our construction.

- The unique smallest odd cycle transversal of $A(u, v)$ is $\left\{a_{1}, a_{3}\right\}$. We call this the passive odd cycle transversal of the arrow.

- In $A(u, v) \backslash\left\{a_{1}, a_{3}\right\}, u$ and $v$ are in different connected components.

- The set $\left\{a_{2}, v\right\}$ is a smallest odd cycle transversal of $A(u, v) \backslash\{u\}$. We call this the active odd cycle transversal of the arrow.

The intuition behind an arrow from $u$ to $v$ is that if $u$ is put into the odd cycle transversal, then $v$ can be put into the odd cycle transversal "for free." When the active odd cycle transversal of the arrow is picked, we say the arrow is active; otherwise, we say the arrow is passive.

To construct $G$, we make $t \cdot p$ paths, $\left\{P_{i, j}\right\}$ for $1 \leq i \leq t, 1 \leq j \leq p$ (see Figure 8 ). Each path has $3 m(t p+1)$ vertices, and the vertices of $P_{i, j}$ are denoted by $p_{i, j}^{\ell}$ for $1 \leq \ell \leq 3 m(t p+1)$. For a fixed $i$, the paths $\left\{P_{i, j}: 1 \leq j \leq p\right\}$ correspond to the set $F_{i}$ of variables. For every $1 \leq i \leq t, 1 \leq j \leq p$, and $1 \leq \ell<3 m(t p+1)$, we add three vertices $a_{i, j}^{\ell}, b_{i, j}^{\ell}$ and $q_{i, j}^{\ell}$ adjacent to each other. We also add the edges $a_{i, j}^{\ell} p_{i, j}^{\ell}$ and $b_{i, j}^{\ell} p_{i, j}^{\ell+1}$. One can think of the vertices of the paths $\left\{P_{i, j}\right\}$ layed out as rows in a matrix, where for every fixed $1 \leq \ell \leq 3 m(t p+1)$ there is a column $\left\{p_{i, j}^{\ell}: 1 \leq i \leq t, 1 \leq\right.$ $j \leq p\}$. We group the colums three by three. In particular, for every $i \leq t$ and $0 \leq \ell<m(t p+1)$, we define the sets $P_{i}^{\ell}=\left\{p_{i, j}^{3 \ell+1}, p_{i, j}^{3 \ell+2}, p_{i, j}^{3 \ell+3}: 1 \leq j \leq p\right\}, A_{i}^{\ell}=\left\{a_{i, j}^{3 \ell+1}, a_{i, j}^{3 \ell+2}, a_{i, j}^{3 \ell+3}: 1 \leq j \leq p\right\}, B_{i}^{\ell}=$ $\left\{b_{i, j}^{3 \ell+1}, b_{i, j}^{3 \ell+2}, b_{i, j}^{3 \ell+3}: 1 \leq j \leq p\right\}$ and $Q_{i}^{\ell}=\left\{q_{i, j}^{3 \ell+1}, q_{i, j}^{3 \ell+2}, q_{i, j}^{3 \ell+3}: 1 \leq j \leq p\right\}$.

For every $i \leq t$ and $0 \leq \ell<m(t p+1)$, we make two new sets $L_{i}^{\ell}$ and $R_{i}^{\ell}$ of new vertices. Both $L_{i}^{\ell}$ and $R_{i}^{\ell}$ are independent sets of size $5 p$, and we add all the edges possible between $L_{i}^{\ell}$ and $R_{i}^{\ell}$. From $L_{i}^{\ell}$, we pick a special vertex $\lambda_{i}^{\ell}$ and from $R_{i}^{\ell}$, we pick $\rho_{i}^{\ell}$. We make all the vertices in $A_{i}^{\ell}$ adjacent to 

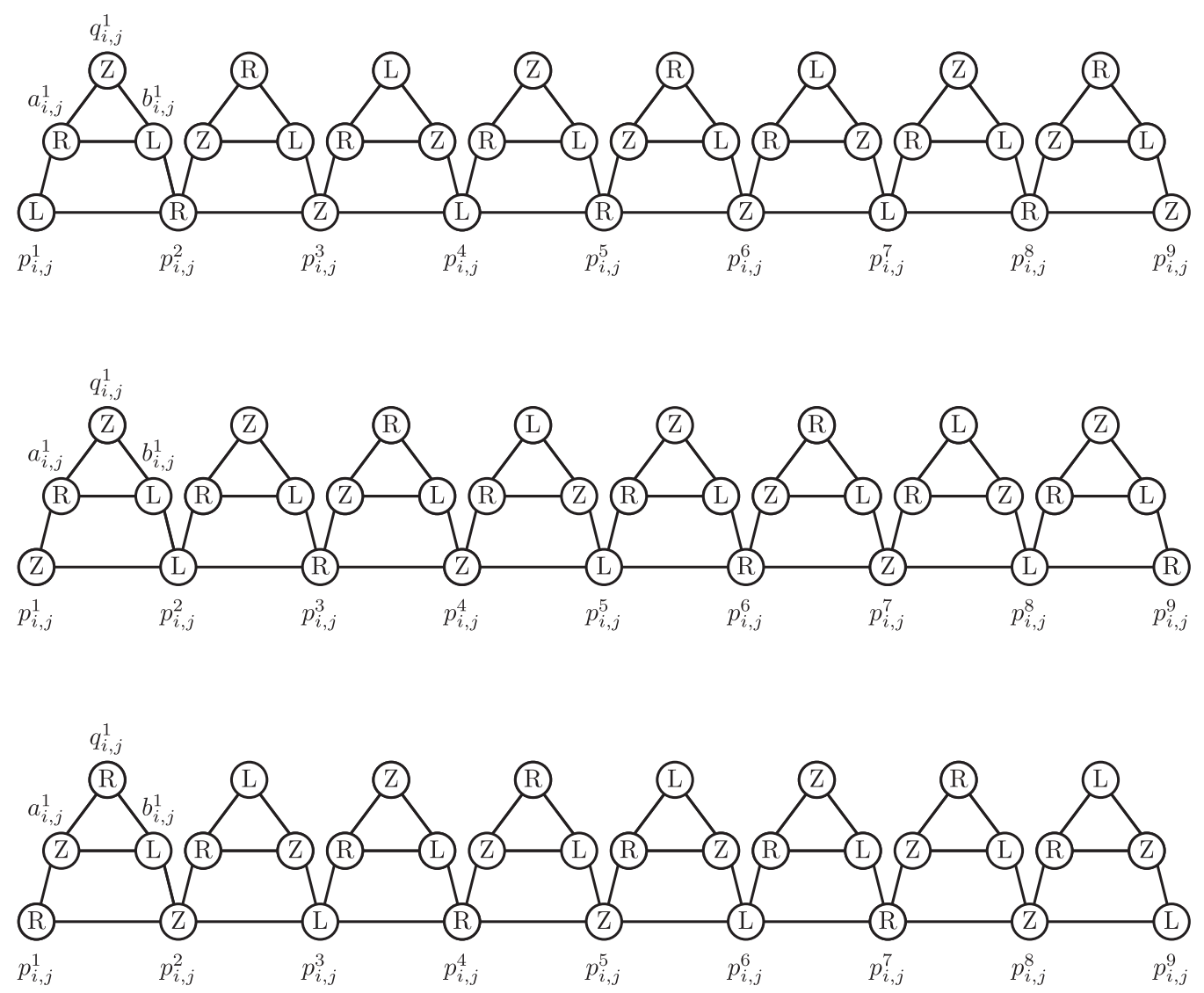

Fig. 8. Reduction to Odd Cycle Transversal. The path $P_{i, j}$ with three different ways of removing a set $Z$ and partitioning the remaining bipartite graph into classes $L$ and $R$.

all vertices of $L_{i}^{\ell}$, and we make all vertices in $B_{i}^{\ell}$ adjacent to all vertices of $R_{i}^{\ell}$. We make $\lambda_{i}^{\ell}$ adjacent to $\rho_{i}^{\ell+1}$, except for $\ell=m(t p+1)-1$.

We will say that a subset $S$ of $P_{i}^{\ell}$ that picks exactly one vertex from $P_{i, j}$ for every $1 \leq j \leq p$ is good. The idea is that there are $3^{p} \geq 2^{h}$ good subsets of $P_{i}^{\ell}$, so we can make group assignments of $F_{i}$ correspond to good subsets of $P_{i}^{\ell}$. For every good subset $S$ of $P_{i}^{\ell}$, we add a cycle $X_{i, S}^{\ell}$. The cycle $X_{i, S}^{\ell}$ has length $2 p+1$. We select a vertex on $X_{i, S}^{\ell}$ and call it $x_{i, S}^{\ell}$. For every vertex $u \in P_{i}^{\ell} \backslash S$, we add an arrow from $u$ to a vertex of $X_{i, S}^{\ell}$. We add arrows in such a way that every vertex of $X_{i, S}^{\ell} \backslash\left\{x_{i, S}^{\ell}\right\}$ is the endpoint of exactly one arrow.

For every $i \leq t$ and $0 \leq \ell<m(t p+1)$, we make a cycle $Y_{i}^{\ell}$ of length $3^{p}$. Notice that the length of the cycle is odd. Every vertex of $Y_{i}^{\ell}$ corresponds to a good subset $S$ of $P_{i}^{\ell}$. For each good subset $S$ of $P_{i}^{\ell}$, we add an arrow from $x_{i, S}^{\ell}$ of the cycle $X_{i, S}^{\ell}$ to the vertex in $Y_{i}^{\ell}$ that corresponds to $S$.

We say that a good subset of $P_{i}^{\ell}$ is equal with a good subset $S^{\prime}$ of $P_{i}^{\ell^{\prime}}$ if for every $1 \leq j \leq t$, the distance along $P_{i, j}$ between the vertex of $S$ on $P_{i, j}$ and the vertex of $S^{\prime}$ on $P_{i, j}$ is divisible by 3 . Informally, $S$ and $S^{\prime}$ are equal if they look identical when we superimpose $P_{i}^{\ell}$ onto $P_{i}^{\ell^{\prime}}$. To every group assignment of variables $F_{i}$, we designate a good subset of $P_{i}^{\ell}$ for every $\ell$. We designate good subsets in such a way that good subsets corresponding to the same group assignment are equal. 
Finally, for every clause $C_{j}, 1 \leq j \leq m$, we will introduce $t p+1$ cycles. That is, for every $0 \leq r \leq$ $t p$, we inroduce a cycle $\widehat{C}_{j}^{r}$. The cycle contains one vertex for every $i \leq t$ and group assignment to $F_{i}$, and potentially one dummy vertex to make it have odd length. Going around the cycle counterclockwise, we first encounter all the vertices corresponding to group assignments of $F_{1}$, then all the vertices corresponding to group assignments of $F_{2}$, and so on. For $i \leq t$ and every good subset $S$ of $P_{i}^{r m+j}$ that corresponds to a group assignment of $F_{i}$ that satisfies $C_{j}$, we add an arrow from $x_{i, S}^{r m+j}$ to the vertex on $\widehat{C}_{j}^{r}$ that corresponds to the same group assignment of $F_{i}$ as $S$ does. This concludes the construction of $G$.

The intention behind the construction is that if $\phi$ is satisfiable, then a minimum odd cycle transversal of $G$ can pick:

- One vertex from each triangle $\left\{a_{i, j}^{\ell}, b_{i, j}^{\ell}, q_{i, j}^{\ell}\right\}$ for each $1 \leq i \leq t, 1 \leq j \leq p, 1 \leq \ell<3 m(t p+$ $1)$. There are $t p(3 m(t p+1)-1)$ such triangles in total.

- One vertex from $\left\{p_{i, j}^{3 \ell+1}, p_{i, j}^{3 \ell+2}, p_{i, j}^{3 \ell+3}\right\}$ for each $1 \leq i \leq t, 1 \leq j \leq p, 0 \leq \ell<m(t p+1)$. There are $t p m(t p+1)$ such triples.

- Two vertices from every arrow added, without counting the starting point of the arrow. For each $i \leq t$ and $0 \leq \ell<m(t p+1)$, there are $2 p 3^{p}$ arrows ending in some cycle $X_{i, S}^{\ell}$. Hence, there are $2 p 3^{p} t m(t p+1)$ such arrows. For every $i \leq t$ and $0 \leq \ell<m(t p+1)$ there are $3^{p}$ arrows ending in the cycle $Y_{i}^{\ell}$. Hence, there are $3^{p} t m(t p+1)$ such arrows. For every clause $C_{j}$, there are $t p+1$ arrows added for every group assignment that satisfies that clause. Let $\mu$ be the sum over all clauses of the number of group assignments that satisfy that clause. The total number of arrows added is then $\mu(t p+1)+(2 p+1) 3^{p} t m(t p+1)$. Thus, the odd cycle transversal can pick $2 \mu(t p+1)+2(2 p+1) 3^{p} t m(t p+1)$ vertices from arrows.

- One vertex $x_{i, S}^{\ell}$ for every $i \leq t$ and $0 \leq \ell<m(t p+1)$. There are $t m(t p+1)$ choices for $i$ and $\ell$.

We let the $\alpha$ be the value of the total budget, that is the sum of the items above.

LEMMA 15. If $\phi$ is satisfiable, then $G$ has an odd cycle transversal of size $\alpha$.

Proof. Given a satisfying assignment $\gamma$ to $\phi$, we construct an odd cycle transversal $Z$ of $G$ of size $\alpha$ together with a partition of $V(G) \backslash Z$ into $L$ and $R$ such that every edge of $G \backslash Z$ goes between a vertex in $L$ and a vertex in $R$. The assignment to $\phi$ corresponds to a group assignment of each $F_{i}$ for $1 \leq i \leq t$. For every $1 \leq i \leq t$ and $0 \leq \ell<m(t p+1)$, we add to $Z$ the good subset $S$ of $P_{i}^{\ell}$ that corresponds to the group assignment of $F_{i}$. Notice that for each fixed $i$, the sets picked from $P_{i}^{\ell}$ and $P_{i}^{\ell^{\prime}}$ are equal for any $\ell, \ell^{\prime}$. At this point, we have picked one vertex from $\left\{p_{i, j}^{3 \ell+1}, p_{i, j}^{3 \ell+2}, p_{i, j}^{3 \ell+3}\right\}$ for each $1 \leq i \leq t, 1 \leq j \leq p, 0 \leq \ell<m(t p+1)$.

For every fixed $1 \leq i \leq t, 1 \leq j \leq p$, there are three cases. If $p_{i, j}^{1} \in Z$, then we put $p_{i, j}^{2}$ into $L$ and $p_{i, j}^{3}$ into $R$. If $p_{i, j}^{2} \in Z$, then we put $p_{i, j}^{1}$ into $R$ and $p_{i, j}^{3}$ into $L$. If $p_{i, j}^{3} \in Z$, then we put $p_{i, j}^{1}$ into $L$ and $p_{i, j}^{2}$ into $R$. Now, for every $4 \leq \ell \leq 3 m(t p+1)$ such that $p_{i, j}^{\ell} \notin Z$, we put $p_{i, j}^{\ell}$ into the same set out of $\{L, R\}$ as $p_{i, j}^{\ell^{\prime}}$ where $1 \leq \ell^{\prime} \leq 3$ and $\ell \equiv \ell^{\prime} \bmod 3$.

For every $1 \leq i \leq t, 0 \leq \ell \leq m(t p+1)$, we put $L_{i}^{\ell}$ into $L$ and $R_{i}^{\ell}$ into $R$. For every triple of $a, b, q$ of pairwise adjacent vertices such that $a \in A_{i}^{\ell}, b \in B_{i}^{\ell}$, and $q \in Q_{i}^{\ell}$, we proceed as follows. The vertex $a$ has a neighbour $a^{\prime}$ in $P_{i}^{\ell}$ and $b$ has a neighbour $b^{\prime}$ in $P_{i}^{\ell}$. There is a $j$ such that $b^{\prime}$ is the successor of $a^{\prime}$ on $P_{i, j}$. Thus, there are three cases:

- $a^{\prime} \in Z$ and $b^{\prime} \in L$, we put $a$ in $R, q$ in $L$ and $b$ in $Z$;

- $a^{\prime} \in R$ and $b^{\prime} \in Z$, we put $a$ in $Z, q$ in $R$ and $b$ in $L$;

- $a^{\prime} \in L$ and $b^{\prime} \in R$, we put $a$ in $R, q$ in $Z$ and $b$ in $L$. 
For every $1 \leq i \leq t, 0 \leq \ell \leq m(t p+1)$, there are many arrows from vertices in $P_{i}^{\ell}$ to vertices on cycles $X_{i, S}^{\ell}$ for good subsets $S$ of $P_{i}^{\ell}$. For each arrow, if its endpoint in $P_{i}^{\ell}$ is in $Z$, we add the active odd cycle transversal of the arrow to $Z$, otherwise, we add the passive odd cycle transversal of the arrow to $Z$. In either case, the remaining vertices on the arrow form a forest, and therefore we can insert the remaining vertices of the arrow into $L$ and $R$ according to which sets out of $\{L, R, Z\} u$ and $v$ are in.

For every $1 \leq i \leq t, 0 \leq \ell \leq m(t p+1)$, there is exactly one set $S$ such that the cycle $X_{i, S}^{\ell}$ only has passive arrows pointing into it. This is exactly the set $S$, which corresponds to the restriction of $\gamma$ to $F_{i}$. Each cycle $X_{i, S^{\prime}}^{\ell}$ that has at least one arrow pointing into them already contains at least one vertex in $Z$-the endpoint of the active arrow pointing into the cycle. Thus, we can partition the remaining vertices of $X_{i, S^{\prime}}^{\ell}$ into $L$ and $R$ such that no edge has both endpoints in $L$ or both endpoints in $R$. For the cycle $X_{i, S}^{\ell}$, we put $x_{i, S}^{\ell}$ into $Z$ and partition the remaining vertices of $X_{i, S}^{\ell}$ into $L$ and $R$ such that no edge has both endpoints in $L$ or both endpoints in $R$. We add the active odd cycle transversal in the arrow from $x_{i, S}^{\ell}$ to the cycle $Y_{i}^{\ell}$ into $Z$. For all other good subsets $S^{\prime}$, we add the passive odd cycle transversal in the arrow from $x_{i, S}^{\ell}$ to the cycle $Y_{i}^{\ell}$ into $Z$. Thus, each cycle $Y_{i}^{\ell}$ contains one vertex in $Z$ and the remaining vertices of $Y_{i}^{\ell}$ can be distributed into $L$ and $R$.

For every arrow that goes from a vertex $x_{i, S}^{\ell}$ into a cycle $\widehat{C}_{h}^{r}$, we add the active odd cycle transversal of the arrow to $Z$ if $x_{i, S}^{\ell} \in Z$ and add the passive odd cycle transversal to $Z$, otherwise. Again the remaining vertices on each arrow can easily be partitioned into $L$ and $R$ such that no edge has both endpoints in $L$ or both endpoints in $R$. This concludes the construction of $Z$. Since we have put the vertices into $Z$ in accordance to the budget described in the construction it follows that $|Z| \leq \alpha$. All that remains to show, is that for each $1 \leq h \leq m$ and $0 \leq r \leq t p$, the cycle $\widehat{C}_{h}^{r}$ has at least one active arrow pointing into it.

The cycle $\widehat{C}_{h}^{r}$ corresponds to the clause $C_{h}$. The clause $C_{h}$ is satisfied by $\gamma$, and hence it is satisfied by the restriction of $\gamma$ to some group $F_{i}$. This restriction is a group assignment of $F_{i}$, and hence it corresponds to a good subset $S$ of $P_{i}^{r m+h}$, which happens to be exactly $Z \cap P_{i}^{r m+h}$. Thus, $x_{i, S}^{r m+h} \in Z$, and since the restriction of $\gamma$ to $F_{i}$ satisfies $C_{h}$, there is an arrow pointing from $x_{i, S}^{r m+h}$ and into $\widehat{C}_{h}^{r}$. Since this arrow is active, this concludes the proof.

LEMmA 16. If $G$ has an odd cycle transversal of size $\alpha$, then $\phi$ is satisfiable.

Proof. Let $Z$ be an odd cycle transversal of $G$ of size $\alpha$. Since $G \backslash Z$ is bipartite, the vertices of $G \backslash Z$ can be partitioned into $L$ and $R$ such that every edge of $G \backslash Z$ has one endpoint in $L$ and the other in $R$. Given $Z, L$, and $R$, we construct a satisfying assignment to $\phi$. Every arrow in $G$ must contain at least two vertices in $Z$, not counting the startpoint of the arrow. Let $\vec{Z}$ be a subset of $Z$ containing two vertices from each arrow, but no arrow start point. Observe that no two arrows have the same endpoint, and therefore $|\vec{Z}|$ is exactly two times the number of arrows in $G$. Let $Z^{\prime}=Z \backslash \vec{Z}$.

We argue that for any $1 \leq i \leq t$ and $0 \leq \ell<m(t p+1)$, we have $\mid Z^{\prime} \cap\left(L_{i}^{\ell} \cup R_{i}^{\ell} \cup A_{i}^{\ell} \cup B_{i}^{\ell} \cup Q_{i}^{\ell} \cup\right.$ $\left.P_{i}^{\ell}\right) \mid \geq 4 p$. Observe that no vertices in $L_{i}^{\ell}, R_{i}^{\ell}, A_{i}^{\ell}, B_{i}^{\ell}, Q_{i}^{\ell}$, or $P_{i}^{\ell}$ are endpoints of arrows, and hence they do not contain any vertices of $\vec{Z}$. Suppose for contradiction that $\mid Z^{\prime} \cap\left(L_{i}^{\ell} \cup R_{i}^{\ell} \cup A_{i}^{\ell} \cup B_{i}^{\ell} \cup\right.$ $\left.Q_{i}^{\ell} \cup P_{i}^{\ell}\right) \mid<4 p$. Then, there is a vertex in $\lambda \in L_{i}^{\ell} \backslash Z^{\prime}$ and a vertex $\rho \in R_{i}^{\ell} \backslash Z^{\prime}$. Without loss of generality, $\lambda \in L$ and $\rho \in R$. Furthermore, there is a $1 \leq j \leq p$ such that

$$
\left|Z^{\prime} \cap\left\{p_{i, j}^{3 \ell+1}, p_{i, j}^{3 \ell+2}, p_{i, j}^{3 \ell+3}, a_{i, j}^{3 \ell+1}, a_{i, j}^{3 \ell+2}, a_{i, j}^{3 \ell+3}, b_{i, j}^{3 \ell+1}, b_{i, j}^{3 \ell+2}, b_{i, j}^{3 \ell+3}, q_{i, j}^{3 \ell+1}, q_{i, j}^{3 \ell+2}, q_{i, j}^{3 \ell+3}\right\}\right|<4 .
$$

Since $\left\{a_{i, j}^{3 \ell+1}, b_{i, j}^{3 \ell+1}, q_{i, j}^{3 \ell+1}\right\},\left\{a_{i, j}^{3 \ell+2}, b_{i, j}^{3 \ell+2}, q_{i, j}^{3 \ell+2}\right\}$, and $\left\{a_{i, j}^{3 \ell+3}, b_{i, j}^{3 \ell+3}, q_{i, j}^{3 \ell+3}\right\}$ form triangles and must contain a vertex from $Z^{\prime}$ each, it follows that each of these triangles contain exactly one vertex 
from $Z^{\prime}$ and that $Z^{\prime} \cap\left\{p_{i, j}^{3 \ell+1}, p_{i, j}^{3 \ell+2}, p_{i, j}^{3 \ell+3}\right\}=\emptyset$. Since $\lambda \in L$ and $\rho \in R, \lambda$ is adjacent to all vertices of $A_{i, j}^{\ell}$ and $\rho$ is adjacent to all vertices of $B_{i, j}^{\ell}$, it follows that $A_{i, j}^{\ell} \backslash Z^{\prime} \subseteq R$ and $B_{i, j}^{\ell} \backslash Z^{\prime} \subseteq L$.

Hence, there are two cases to consider either (1) $\left\{p_{i, j}^{3 \ell+1}, p_{i, j}^{3 \ell+3}\right\} \subseteq L$ and $p_{i, j}^{3 \ell+2} \in R$ or (2) $\left\{p_{i, j}^{3 \ell+1}, p_{i, j}^{3 \ell+3}\right\} \subseteq R$ and $p_{i, j}^{3 \ell+2} \in L$. In the first case, observe that either $a_{i, j}^{3 \ell+2} \in R$ or $b_{i, j}^{3 \ell+2} \in L$, and hence either $a_{i, j}^{3 \ell+2} p_{i, j}^{3 \ell+2}$ or $b_{i, j}^{3 \ell+2} p_{i, j}^{3 \ell+3}$ have both endpoints in the same set out of $\{L, R\}$, a contradiction. The second case is similar, either $a_{i, j}^{3 \ell+1} \in R$ or $b_{i, j}^{3 \ell+1} \in L$, and hence either $a_{i, j}^{3 \ell+1} p_{i, j}^{3 \ell+1}$ or $b_{i, j}^{3 \ell+1} p_{i, j}^{3 \ell+2}$ have both endpoints in the same set out of $\{L, R\}$, a contradiction. We conclude that $\left|Z^{\prime} \cap\left(L_{i}^{\ell} \cup R_{i}^{\ell} \cup A_{i}^{\ell} \cup B_{i}^{\ell} \cup Q_{i}^{\ell} \cup P_{i}^{\ell}\right)\right| \geq 4 p$.

For any $1 \leq i \leq t$ and $0 \leq \ell<m(t p+1), Y_{i}^{\ell}$ is an odd cycle so $Y_{i}^{\ell}$ contains a vertex in $Z$. If $Y_{i}^{\ell}$ contains no vertices of $Z^{\prime}$, then it contains a vertex from $\vec{Z}$ and there is an active arrow pointing into $Y_{i}^{\ell}$. The starting point of this arrow is a vertex $x_{i, S}^{\ell}$ for some good subset $S$ of $P_{i}^{\ell}$. Since the arrow is active and $x_{i, S}^{\ell}$ is not the endpoint of any arrow, we know that $x_{i, S}^{\ell} \in Z^{\prime}$. Hence, for any $1 \leq i \leq t$ and $0 \leq \ell<m(t p+1)$, we have that either there is a good subset $S$ of $P_{i}^{\ell}$ such that $x_{i, S}^{\ell} \in Z^{\prime}$ or at least one vertex of $Y_{i}^{\ell}$ is in $Z^{\prime}$.

The above arguments, together with the budget constraints, imply that for every $1 \leq i \leq t$ and $0 \leq \ell<m(t p+1)$, we have $\left|Z^{\prime} \cap\left(L_{i}^{\ell} \cup R_{i}^{\ell} \cup A_{i}^{\ell} \cup B_{i}^{\ell} \cup Q_{i}^{\ell} \cup P_{i}^{\ell}\right)\right|=4 p$ and that $\mid Z^{\prime} \cap \bigcup\left\{x_{i, S}^{\ell}\right\} \cup$ $V\left(Y_{i}^{\ell}\right) \mid=1$, where the union is taken over all good subsets $S$ of $P_{i}^{\ell}$. It follows $Z^{\prime} \cap P_{i}^{\ell}$ is a good subset of $P_{i}^{\ell}$. Let $S=Z^{\prime} \cap P_{i}^{\ell}$. The cycle $X_{i, S}^{\ell}$ has odd length, and hence it must contain some vertex from $Z$. On the other hand, all the arrows pointing into $X_{i, S}^{\ell}$ are passive, so $X_{i, S}^{\ell}$ cannot contain any vertices from $\vec{Z}$. Thus $X_{i, S}^{\ell}$ contains a vertex from $Z^{\prime}$, and by the budget constraints this must be $x_{i, S}^{\ell}$.

Now, consider three consecutive vertices $p_{i, j}^{\ell}, p_{i, j}^{\ell+1}, p_{i, j}^{\ell+2}$ for some $1 \leq i \leq t, 1 \leq j \leq p, 1 \leq$ $\ell \leq 3 m(t p+1)-2$. We prove that at least one of them has to be in $Z$. Suppose not. We know that neither $\lambda_{i}^{\lfloor\ell / 3\rfloor}, \rho_{i}^{\lfloor\ell / 3\rfloor}, \lambda_{i}^{\lfloor\ell / 3\rfloor+1}$ nor $\rho_{i}^{\lfloor\ell / 3\rfloor+1}$ are in $Z$. Thus, without loss of generality $\left\{\lambda_{i}^{\lfloor\ell / 3\rfloor}, \lambda_{i}^{\lfloor\ell / 3\rfloor+1}\right\} \subseteq L$ and $\left\{\rho_{i}^{\lfloor\ell / 3\rfloor}, \rho_{i}^{\lfloor\ell / 3\rfloor+1}\right\} \subseteq R$. There are two cases. Either $p_{i, j}^{\ell} \in R$ and $p_{i, j}^{\ell+1} \in L$ or $p_{i, j}^{\ell+1} \in L$ and $p_{i, j}^{\ell+3} \in R$. In the first case, we obtain a contradiction, since either $a_{i, j}^{\ell} \in R$ or $b_{i, j}^{\ell} \in L$. In the second case, we get a contradiction, since either $a_{i, j}^{\ell+1} \in R$ or $b_{i, j}^{\ell+1} \in L$. Hence, for any three consecutive vertices on $P_{i, j}$, at least one of them is in $Z$. Since the budget constraints ensure that there are at most $\left|V\left(P_{i, j}\right)\right| / 3$ vertices in $P_{i, j} \cap Z$, it follows from the pigeon hole principle that there is an $0 \leq r \leq t p$ such that for any $1 \leq i \leq t$ and $1 \leq h \leq m$ and $1 \leq h^{\prime} \leq m$, the set $P_{i}^{r m+h} \cap Z$ equals $P_{i}^{r m+h^{\prime}} \cap Z$. Here equality is in the sense of equality of good subsets of $P_{i}^{\ell}$.

For every $1 \leq i \leq t, P_{i}^{r m+1} \cap Z$ is a good subset of $P_{i}^{r m+1}$. If $P_{i}^{r m+1} \cap Z$ corresponds to a group assignment of $F_{i}$, then we set the variables in $F_{i}$ to this assignment. Otherwise, we set all the variables in $F_{i}$ to false. We need to argue that every clause $C_{h}$ is satisfied by this assignment. Consider the cycle $\widehat{C}_{h}^{r}$. Since it is an odd cycle, it must contain a vertex from $Z$, the budget constraints and the discussion above implies that this vertex is from $\vec{Z}$. Hence, there must be an active arrow pointing into $\widehat{C}_{h}^{r}$. The starting point of this active arrow is a vertex $x_{i, S}^{m r+h}$ for some $i$ and good subset $S$ of $P_{i}^{m r+h}$. The set $S$ corresponds to a group assignment of $F_{i}$ that satisfies $C_{h}$. Since the arrow is active $x_{i, S}^{m r+h} \in Z^{\prime}$, and by the discussion above, we have that $P_{i}^{m r+h} \cap Z^{\prime}=S$. Now, $S=P_{i}^{m r+h} \cap Z^{\prime}$ and $S$ is equal to $P_{i}^{m r+1} \cap Z^{\prime}$, and hence the assignment to the variables of $F_{i}$ satisfies $C_{h}$. Since this holds for all clauses, this concludes the proof.

Lemma 17. $\mathbf{p w}(G) \leq t(p+1)+10 p 3^{p}$. 
Proof. We show how to search the graph using at most $t(p+1)+10 p 3^{p}$ searchers. The strategy consists of $m(t p+1)$ rounds numbered from round 0 to round $m(t p+1)-1$. Each round has $t$ stages, numbered from 1 to $t$. In the beginning of round $k$ there is a searcher on $p_{i, j}^{3 k+1}$ and $\rho_{i}^{k}$ for every $1 \leq i \leq t, 1 \leq j \leq p$. Let $r$ and $1 \leq h \leq m$ be integers such that $k+1=r m+h$. Recall that as we go around $\widehat{C}_{h}^{r}$ counterclockwise, we first encounter vertices corresponding to group assignments of $F_{1}$, then to assignments of $F_{2}$ and so on. In the beginning of round $k$, we place a searcher on the first vertex on $\widehat{C}_{h}^{r}$ that corresponds to an assignment of $F_{1}$. If $\widehat{C}_{h}^{r}$ contains a dummy vertex, then we place a searcher on this vertex as well. These two searchers will remain on their respective vertices throughout the round. In the beginning of stage $s$ of round $k$, we will assume that the vertices on the cycle $\widehat{C}_{h}^{r}$ corresponding to group assignments of $F_{s^{\prime}}, s^{\prime}<s$ have already been cleaned, and in the beginning of every stage $s>1$, there is a searcher standing on the first vertex corresponding to a group assignment of $F_{s}$.

In stage $s$ of round $k$, we place searchers on all vertices of $P_{s}^{k}, A_{s}^{k}, B_{s}^{k}, Q_{s}^{k}, L_{s}^{k}, R_{s}^{k}, Y_{s}^{k}$ and all vertices of cycles $X_{s, S}^{k}$ for every good subset $S$ of $P_{s}^{k}$, on all vertices of arrows starting or ending in such cycles, and on all vertices of $\widehat{C}_{h}^{r}$ corresponding to group assignments of $F_{s}$. In total, this amounts to less than $10 p 3^{p}$ vertices.

In the last part of stage $s$ of round $k$, we place searchers on $p_{s, j}^{3(k+1)+1}$ for every $1 \leq j \leq p$ and on $\rho_{s}^{k+1}$. Then, we remove all the searchers that were placed out in the first part of phase $s$ except for the searcher on the last vertex on $\widehat{C}_{h}^{r}$ corresponding to a group assignment of $F_{s}$. Unless $s=1$ there is also a searcher on the last vertex on $\widehat{C}_{h}^{r}$ corresponding to a group assignment of $F_{s-1}$. We remove this searcher, and the next stage can commence. In the end of the last stage of round $k$, we remove all the searchers from $\widehat{C}_{h}^{r}$. Then, the last stage can commence. At any point in time, at most $t(p+1)+10 p 3^{p}$ searchers are placed on $G$.

Proof (of Theorem 5). Suppose Odd Cycle Transversal can be solved in time $O^{*}((3-$ $\epsilon) \mathbf{p w}(G)$ for some $\epsilon>0$. Then there is an $\epsilon^{\prime}<1$ such that $O^{*}\left((3-\epsilon)^{\mathbf{p w}(G)}\right) \leq O^{*}\left(3^{\epsilon^{\prime} \mathbf{p w}(G)}\right)$. We choose $p$ large enough such that $\epsilon^{\prime} \cdot \frac{p+1}{p-1}=\delta^{\prime}<1$. Given an instance of SAT, we construct an instance of OdD CyCle TrAnsversal using the above construction and the chosen value of $p$. Then, we solve the Odd Cycle Transversal instance using the $O^{*}\left((3-\epsilon)^{\mathbf{p w}(G)}\right)$ time algorithm. Correctness is ensured by Lemmata 15 and 16. Lemma 17 yields that the total time taken is upper bounded by $O^{*}\left((3-\epsilon)^{\mathbf{p w}(G)}\right) \leq O^{*}\left(3^{\epsilon^{\prime} \mathbf{p w}(G)}\right) \leq O^{*}\left(3^{\epsilon^{\prime}\left(t(p+1)+f\left(\epsilon^{\prime}\right)\right)}\right) \leq O^{*}\left(3^{\epsilon^{\prime}\left\lceil\frac{n}{[p \log 3]}\right\rceil(p+1)}\right) \leq$ $O^{*}\left(3^{\epsilon^{\prime} \frac{n(p+1)}{[p \log 3\rfloor}}\right) \leq O^{*}\left(3^{\epsilon^{\prime} \frac{n(p+1)}{(p-1) \log 3}}\right) \leq O^{*}\left(3^{\delta^{\prime} \frac{n}{\log 3}}\right) \leq O^{*}\left(2^{\delta^{\prime} n}\right)=. O^{*}\left((2-\delta)^{n}\right)$ for $\delta<1$.

\section{PARTITION INTO TRIANGLES}

A triangle packing in a graph $G$ is a collection of pairwise disjoint vertex sets $S_{1}, S_{2}, \ldots S_{t}$ in $G$ such that $S_{i}$ induces a triangle in $G$ for every $i$. The size of the packing is $t$. If $V(G)=\bigcup_{i \leq t} S_{i}$, then the collection $S_{1} \ldots S_{t}$ is a partition of $G$ into triangles. In the TRIANGLe Packing problem, we are given a graph $G$ and an integer $t$ and asked whether there is a triangle packing in $G$ of size at least $t$. In the Partition Into Triangles problem, we are given a graph $G$ and asked whether $G$ can be partitioned into triangles. Notice that since Partition Into Triangles is the special case of TRIANGLE PACKING when the number of triangles is the number of vertices divided by 3 , the bound of Theorem 6 holds for TRIANgle PACKING as well.

Theorem 6. If Partition Into Triangles can be solved in time $O^{*}\left((2-\epsilon)^{\mathbf{p w}(G)}\right)$ for $\epsilon>0$, then SAT can be solved in $O^{*}\left((2-\delta)^{n}\right)$ time for some $\delta>0$.

Construction. First show the lower bound for Triangle PACKIng and then modify our construction to also work for the more restricted PARTITION INTO TRIANGLEs problem. Given an 


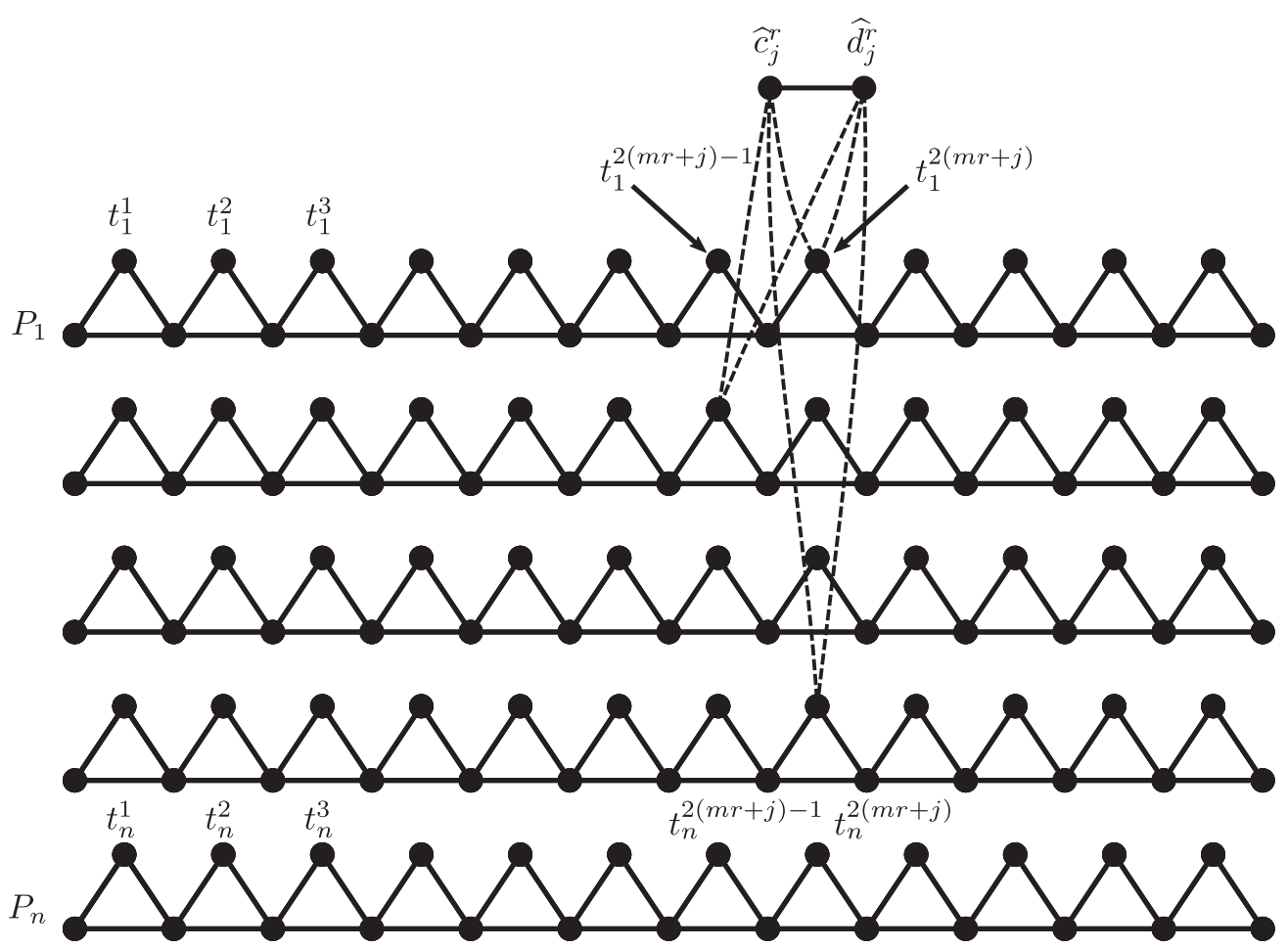

Fig. 9. Reduction to TRIANGle PACkING, showing how the vertices $\widehat{c}_{j}^{r}$ and $\widehat{d}_{j}^{r}$ representing clause $C_{j}=\left(x_{1} \vee\right.$ $\left.\bar{x}_{2} \vee x_{4}\right)$ are connected to the paths $P_{1}, \ldots, P_{n}$.

instance $\phi$ of SAT, we construct a graph $G$ as follows (see Figure 9). For every variable $v_{i}$, we make a path $P_{i}$ on $2 m(n+1)+1$ vertices. We denote the $\ell$ th vertex of $P_{i}$ by $p_{i}^{\ell}$. For every $i$, we add a set $T_{i}$ of $2 m(n+1)$ vertices, and let the $\ell$ th vertex of $T_{i}$ be denoted $t_{i}^{\ell}$. For every $1 \leq \ell \leq 2 m(n+1)$, we add the edges $t_{i}^{\ell} p_{i}^{\ell}$ and $t_{i}^{\ell} p_{i}^{\ell+1}$.

For every clause $C_{j}$, we add $n+1$ gadgets corresponding to the clause. In particular, for every $0 \leq$ $r \leq n$, we do the following. First, we add the vertices $\widehat{c}_{j}^{r}$ and $\widehat{d}_{j}^{r}$ and the edge $\widehat{c}_{j}^{r} \widehat{d}_{j}^{r}$. For every variable $v_{i}$ that occurs in $C_{j}$ positively, we add the edges $\widehat{c}_{j}^{r} t_{i}^{2(m r+j)}$ and $\widehat{d}_{j}^{r} t_{i}^{2(m r+j)}$. For every variable $v_{i}$ that occurs in $C_{j}$ negated, we add the edges $\widehat{c}_{j}^{r} t_{i}^{2(m r+j)-1}$ and $\widehat{d}_{j}^{r} t_{i}^{2(m r+j)-1}$. Doing this for every $r$ and every clause $C_{j}$ concludes the construction of $G$.

Lemma 18. If $\phi$ satisfiable, then $G$ has a triangle packing of size $m n(n+1)+m(n+1)$.

Proof. Consider a satisfying assignment to $\phi$. For every variable $v_{i}$ that is set to true and integer $1 \leq \ell \leq m(n+1)$, we add $\left\{t_{i}^{2 l-1}, p_{i}^{2 l-1}, p_{i}^{2 l}\right\}$ to the triangle packing. For every variable $v_{i}$ that is set to false and integer $1 \leq \ell \leq m(n+1)$, we add $\left\{t_{i}^{2 l}, p_{i}^{2 l}, p_{i}^{2 l+1}\right\}$ to the triangle packing. For every clause $C_{j}$, there is a literal set to true. Suppose this literal corresponds to the variable $v_{i}$. Notice that if $v_{i}$ occurs positively in $C_{j}$, then $v_{i}$ is set to true, and if it occurs negatively it is set to false. For each $0 \leq r \leq n$, if $v_{i}$ occurs positively in $C_{j}$, then $t_{i}^{2(m r+j)}$ has not yet been used in any triangle, so we can add $\left\{\widehat{c}_{j}^{r}, \widehat{d}_{j}^{r}, t_{i}^{2(m r+j)}\right\}$ to the triangle packing. On the other hand, if $v_{i}$ occurs negated in 
$C_{j}$, then $t_{i}^{2(m r+j)-1}$ has not yet been used in any triangle, so we can add $\left\{\widehat{c}_{j}^{r}, \widehat{d}_{j}^{r}, t_{i}^{2(m r+j)-1}\right\}$ to the triangle packing. In total $m n(n+1)+m(n+1)$ triangles are packed.

LEMMA 19. If $G$ has a triangle packing of size $m n(n+1)+m(n+1)$, then $\phi$ satisfiable.

Proof. Observe that for any $j$ and $r$, every triangle that contains $\widehat{c}_{j}^{r}$ also contains $\widehat{d}_{j}^{r}$ and vice versa. Furthermore, if we remove all the vertices $\widehat{c}_{j}^{r}$ and $\widehat{d}_{j}^{r}$ for every $j$ and $r$ from $G$, we obtain a disconnected graph with $n$ connected components, $G\left[T_{i} \cup V\left(P_{i}\right)\right]$ for every $i$. Thus, the only way to pack $m n(n+1)+m(n+1)$ triangles in $G$ is to pack $m n(n+1)$ triangles in each component $G\left[T_{i} \cup V\left(P_{i}\right)\right]$ and in addition make sure that every pair $\left(\widehat{c}_{j}^{r}, \widehat{d}_{j}^{r}\right)$ is used in some triangle in the packing.

The only way to pack $m n(n+1)$ triangles in a component $G\left[T_{i} \cup V\left(P_{i}\right)\right]$ is to use every second triangle of the form $\left\{t_{i}^{\ell}, p_{i}^{\ell}, p_{i}^{\ell+1}\right\}$, except possibly at one point where two triangles on this form are skipped. By the pigeon hole principle there is an $0 \leq r \leq n$ such that for every $i$, every second triangle of the form $\left\{t_{i}^{2 m r+\ell}, p_{i}^{2 m r+\ell}, p_{i}^{2 m r+\ell+1}\right\}$ for $1 \leq \ell \leq 2 m$ is used. We make an assignment to the variables of $\phi$ as follows. For every $i$ such that $\left\{t_{i}^{2 m r+1}, p_{i}^{2 m r+1}, p_{i}^{2 m r+2}\right\}$ is used, $v_{i}$ is set to true, and otherwise $\left\{t_{i}^{2 m r+2}, p_{i}^{2 m r+2}, p_{i}^{2 m r+3}\right\}$ is used in the packing and $v_{i}$ is set to false. We prove that this assignment satisfies $\phi$.

For every $j$, the pair $\left(\widehat{c}_{j}^{r}, \widehat{d}_{j}^{r}\right)$ is used in some triangle in the packing. This triangle either contains $t_{i}^{2(m r+j)}$ or $t_{i}^{2(m r+j)-1}$ for some $i$. If it contains $t_{i}^{2(m r+j)}$, then $v_{i}$ occurs positively in $C_{j}$. Furthermore, since the triangle packing contains every second triangle of the form $\left\{t_{i}^{2 m r+\ell}, p_{i}^{2 m r+\ell}, p_{i}^{2 m r+\ell+1}\right\}$ for $1 \leq \ell \leq 2 m$, it follows that the triangle packing contains $\left\{t_{i}^{2 m r+1}, p_{i}^{2 m r+1}, p_{i}^{2 m r+2}\right\}$, and hence $v_{i}$ is set to true. By an identical argument, if the triangle containing the pair $\left(\widehat{c}_{j}^{r}, \widehat{d}_{j}^{r}\right)$ contains $t_{i}^{2(m r+j)-1}$, then $v_{i}$ occurs negated in $C_{j}$ and $v_{i}$ is set to false. This concludes the proof.

We now modify the construction to work for PARTition Into Triangles instead of Triangle PACKIng. Given the graph $G$ as constructed from $\phi$, we construct a graph $G^{\prime}$ as follows. For every $1 \leq i \leq n$ and $1 \leq l \leq m(n+1)$, we make a clique $Q_{i}^{\ell}$ on four vertices. The vertices of $Q_{i}^{\ell}$ are all adjacent to $t_{i}^{2 l}$ and to $t_{i}^{2 l-1}$. For every $i<n$ and $1 \leq l \leq m(n+1)$, we make all vertices of $Q_{i}^{\ell}$ adjacent to all vertices of $Q_{i+1}^{\ell}$. Suppose that $2 n+2$ is $p$ modulo 3 for some $p \in\{0,1,2\}$. We remove $p$ vertices from $Q_{n}^{\ell}$ for every $l \leq m(n+1)$.

Lemma 20. $G$ has a triangle packing of size $\alpha$ if and only if $G^{\prime}$ can be partitioned into triangles. Here, $\alpha$ is a non-negative integer.

Proof. In the forward direction, consider a triangle packing of size $\alpha$ in $G$ as constructed in Lemma 18. We can assume that the triangle packing has this form, because by Lemma 19, we have that $\phi$ is satisfiable.

For every fixed $1 \leq l \leq m(n+1)$, we proceed as follows. We know that there exists an $i$ such that both $t_{i}^{2 l}$ and $t_{i}^{2 l-1}$ are used in the packing. For every $i^{\prime} \neq i$, exactly one out of $t_{i^{\prime}}^{2 l}$ and $t_{i^{\prime}}^{2 l-1}$ is used in the packing. For each such $i^{\prime}$, we make a triangle containing the unused vertex out of $t_{i^{\prime}}^{2 l}$ and $t_{i^{\prime}}^{2 l-1}$ and two vertices of $Q_{i^{\prime}}^{\ell}$. Then, we "clean up" $Q_{1}^{\ell}, \ldots, Q_{n}^{\ell}$ as follows.

In particular, we start with the yet unused vertices of $Q_{1}^{\ell}$. There are two of them. Make a triangle containing these two vertices and one vertex of $Q_{2}^{\ell}$. Now $Q_{2}^{\ell}$ has one unused vertex left. Make a triangle containing this vertex and the two unused vertices of $Q_{3}^{\ell}$. Continue in this fashion until arrive at $Q_{i^{\prime}}^{\ell}$. At this point, we have used 0,1 , or 2 vertices of $Q_{i^{\prime}}^{\ell}$ a triangle containing some vertices in $Q_{i^{\prime}-1}^{\ell}$. The case when we have used 0 vertices of $Q_{i^{\prime}}^{\ell}$ also covers the case that $i^{\prime}=1$. If we only used 0 or 1 vertices of $Q_{i^{\prime}}^{\ell}$, then we add a triangle that contains 3 vertices of $Q_{i^{\prime}}^{\ell}$. If there are still 
unused vertices in $Q_{i^{\prime}}^{\ell}$, then their number is either 1 or 2 . We make a triangle containing these vertices and 1 or 2 of the unused vertices of $Q_{i^{\prime}+1}^{\ell}$. Now, we proceed to $Q_{i^{\prime}+1}^{\ell}$ and continue in this manner until we reach $Q_{n}^{\ell}$. Since the total number of vertices in $\bigcup_{j \leq n} Q_{j}^{\ell}$ is $4 n-p$, we know that $2 n-2$ of these vertices are used for triangles with vertices of $G$, and $2 n+2-p$ is divisible by 3 the process described above will partition all the unused vertices of $\bigcup_{j \leq n} Q_{j}^{\ell}$ into triangles.

In the reverse direction, we argue that in any partitioning of $G^{\prime}$ into triangles, exactly $\alpha$ triangles must lie entirely within $G$. In fact, we argue that for any $l \leq m(n+1)$ exactly $n-1$ vertices out of $\bigcup_{i \leq n}\left\{t_{i}^{2 l}, t_{i}^{2 l-1}\right\}$ are used in triangles containing vertices from $\bigcup_{i \leq n} Q_{i}^{\ell}$.

Pick $1 \leq j \leq m$ and $r$ such that $l=m r+j$. Exactly one out of $\bigcup_{i \leq n}\left\{t_{i}^{2 l}, t_{i}^{2 l-1}\right\}$ is in a triangle with $\widehat{c}_{j}^{r}$ and $\widehat{d}_{j}^{r}$. Furthermore, for each $i \leq n$ the vertex $p_{i}^{2 l}$ must be in a triangle either containing $t_{i}^{2 l}$ or $t_{i}^{2 l}$. Hence, at most $n-1$ vertices out of $\bigcup_{i \leq n}\left\{t_{i}^{2 l}, t_{i}^{2 l-1}\right\}$ are used in triangles containing vertices from $\bigcup_{i \leq n} Q_{i}^{\ell}$. Furthermore, any triangle containing $t_{i}^{2 l}$ or $\left.t_{i}^{2 l-1}\right\}$ must either contain $p_{i}^{2 l}, \widehat{c}_{j}^{r}$ or some vertex in $\bigcup_{i \leq n} Q_{i}^{\ell}$. Hence, exactly $n-1$ vertices out of $\bigcup_{i \leq n}\left\{t_{i}^{2 l}, t_{i}^{2 l-1}\right\}$ are used in triangles containing vertices from $\bigcup_{i \leq n} Q_{i}^{\ell}$. Thus in the packing, exactly $3 \alpha$ vertices in $G^{\prime}$ are contained in triangles completely inside $G$, and hence $G$ has a triangle packing of size $\alpha$.

To complete the proof for Partition Into Triangles, we need to bound the pathwidth of $G^{\prime}$.

LEMMA 21. $\mathbf{p w}\left(G^{\prime}\right) \leq n+10$.

Proof. We give a search strategy for $G^{\prime}$ that uses $n+10$ searchers. The strategy consists of $m(n+1)$ rounds and each round has $n$ stages. In the beginning of round $l, 1 \leq l \leq m(n+1)$, there are $n$ searchers placed, one on each vertex $p_{i}^{2 l-1}$ for every $i$. Let $r$ and $1 \leq j \leq m$ be integers such that $l=m r+j$. We place one searcher on $\widehat{c}_{j}^{r}$ and one on $\widehat{d}_{j}^{r}$. These two searchers will stay put throughout the duration of this round. In stage $i$ of round $l$, we place searchers on all vertices of $Q_{i}^{\ell}$ and $Q_{i+1}^{\ell}$. Then, we place searchers on $t_{i}^{2 l-1}, t_{i}^{2 l}, p_{i}^{2 l}$ and $p_{i}^{2 l+1}$. At the end of stage $i$, we remove the searchers from $Q_{i}^{\ell}, t_{i}^{2 l-1}, t_{i}^{2 l}$ and $p_{i}^{2 l}$. We then proceed to the next stage. At the end of the round, we remove the searchers from $\widehat{c}_{j}^{r}$ and $\widehat{d}_{j}^{r}$. Notice that now there are searchers on $p_{i}^{2 l+1}$ for every $i$, and the next round can commence.

Lemmata 18, 19, 20, and 21 prove Theorem 6.

\section{CONCLUSION}

We have showed that for a number of basic graph problems, the best-known algorithms parameterized by treewidth are optimal in the sense that base of the exponential dependence on treewidth is best possible. Recall that for Dominating Set and Partition Into Triangles, this running time was obtained using the technique of fast subset sum convolutions [50]. Thus, it could have been a real possibility that the running time is improved for some other problems as well.

The results are proved under the Strong Exponential Time Hypothesis (SETH). While this hypothesis is extremely strong and might not be accepted by everyone, our results at least make a connection between rather specific graph problems and the very basic issue of better SAT algorithms. Our results suggest that one should not try to find better algorithms on bounded treewidth graphs for the problems considered in the article: as this would disprove SETH, such an effort is better spent on trying to disprove SETH directly in the domain of satisfiability. Finally, we suggest the following open questions for future work:

- Can we prove similar tight lower bounds under the restriction that the graph is planar? Or is it possible to find improved algorithms on bounded treewidth planar graphs? 
- For the q-Coloring problem, we were able to prove lower bounds parameterized by the feedback vertex set number. Can we prove such bounds for the other problems as well? Recently, Jaffke and Jansen [33] strengthened our lower bounds for $q$-ColoRING. In particular, they showed that $q$-COLORING parameterized by the modulator to linear forests (a forest where every connected component is a path), say lfvs $(G)$, cannot be solved in time $(q-\epsilon)^{\mathbf{l f v s}(G)}|V(G)|^{O(1)}$.

\section{ACKNOWLEDGMENTS}

We sincerely thank all the reviewers for their insightful comments and suggestions.

\section{REFERENCES}

[1] Amir Abboud, Arturs Backurs, and Virginia Vassilevska Williams. 2015. Tight hardness results for LCS and other sequence similarity measures. In Proceedings of the 56th Annual IEEE Symposium on Foundations of Computer Science (FOCS'15). 59-78.

[2] Amir Abboud and Virginia Vassilevska Williams. 2014. Popular conjectures imply strong lower bounds for dynamic problems. In Proceedings of the 55th Annual IEEE Symposium on Foundations of Computer Science (FOCS'14). 434-443.

[3] Amir Abboud, Virginia Vassilevska Williams, and Huacheng Yu. 2015. Matching triangles and basing hardness on an extremely popular conjecture. In Proceedings of the 47th Annual ACM on Symposium on Theory of Computing (STOC'15). 41-50.

[4] Isolde Adler, Stavros G. Kolliopoulos, Philipp Klaus Krause, Daniel Lokshtanov, Saket Saurabh, and Dimitrios M. Thilikos. 2017. Irrelevant vertices for the planar disjoint paths problem. F. Comb. Theory, Ser. B 122 (2017), 815-843.

[5] Jochen Alber and Rolf Niedermeier. 2002. Improved tree decomposition based algorithms for domination-like problems. In Proceedings of the 5th Latin American Symposium on Theoretical Informatics (LATIN'02) (Lecture Notes in Computer Science), Vol. 2286. Springer, 613-628.

[6] Arturs Backurs and Piotr Indyk. 2015. Edit distance cannot be computed in strongly subquadratic time (unless SETH is false). In Proceedings of the 47th Annual ACM on Symposium on Theory of Computing (STOC'15). 51-58.

[7] MohammadHossein Bateni, Mohammad Taghi Hajiaghayi, and Dániel Marx. 2011. Approximation schemes for steiner forest on planar graphs and graphs of bounded treewidth. J. ACM 58, 5 (2011), 21:1-21:37.

[8] Andreas Björklund, Thore Husfeldt, Petteri Kaski, and Mikko Koivisto. 2007. Fourier meets Möbius: Fast subset convolution. In Proceedings of the 39th Annual ACM on Symposium on Theory of Computing (STOC'07). 67-74.

[9] Ivan Bliznets, Marek Cygan, Pawel Komosa, Lukás Mach, and Michal Pilipczuk. 2016. Lower bounds for the parameterized complexity of minimum fill-in and other completion problems. In Proceedings of the 27th Annual ACM-SIAM Symposium on Discrete Algorithms (SODA'16). 1132-1151.

[10] Hans L. Bodlaender, Marek Cygan, Stefan Kratsch, and Jesper Nederlof. 2015. Deterministic single exponential time algorithms for connectivity problems parameterized by treewidth. Inf. Comput. 243 (2015), 86-111.

[11] Glencora Borradaile and Hung Le. 2016. Optimal dynamic program for $r$-domination problems over tree decompositions. In Proccedings of the 11th International Symposium on Parameterized and Exact Computation, IPEC (LIPIcs), Vol. 63. Schloss Dagstuhl - Leibniz-Zentrum fuer Informatik, 8:1-8:23.

[12] Chris Calabro, Russell Impagliazzo, and Ramamohan Paturi. 2009. The complexity of satisfiability of small depth circuits. In Proceedings of the 4th International Workshop on Parameterized and Exact Computation (IWPEC'09) (Lecture Notes in Computer Science), Vol. 5917. Springer, 75-85.

[13] Shiri Chechik, Daniel H. Larkin, Liam Roditty, Grant Schoenebeck, and Robert Endre Tarjan ands Virginia Vassilevska Williams. 2014. Better approximation algorithms for the graph diameter. In Proceedings of the 25th Annual ACM-SIAM Symposium on Discrete Algorithms (SODA'14). 1041-1052.

[14] Jianer Chen, Xiuzhen Huang, Iyad A. Kanj, and Ge Xia. 2006. On the computational hardness based on linear FPTreductions. f. Comb. Optim. 11, 2 (2006), 231-247.

[15] Jianer Chen, Xiuzhen Huang, Iyad A. Kanj, and Ge Xia. 2006. Strong computational lower bounds via parameterized complexity. f. Comput. Syst. Sci. 72, 8 (2006), 1346-1367.

[16] Radu Curticapean and Dániel Marx. 2016. Tight conditional lower bounds for counting perfect matchings on graphs of bounded treewidth, cliquewidth, and genus. In Proceedings of the 27th Annual ACM-SIAM Symposium on Discrete Algorithms (SODA'16). 1650-1669.

[17] Marek Cygan, Holger Dell, Daniel Lokshtanov, Dániel Marx, Jesper Nederlof, Yoshio Okamoto, Ramamohan Paturi, Saket Saurabh, and Magnus Wahlström. 2016. On problems as hard as CNF-SAT. ACM Trans. Algor. 12, 3 (2016), 41:1-41:24. 
[18] Marek Cygan, Fedor V. Fomin, Alexander Golovnev, Alexander S. Kulikov, Ivan Mihajlin, Jakub Pachocki, and Arkadiusz Socala. 2016. Tight bounds for graph homomorphism and subgraph isomorphism. In Proceedings of the 27th Annual ACM-SIAM Symposium on Discrete Algorithms (SODA'16). 1643-1649.

[19] Marek Cygan, Fedor V. Fomin, Lukasz Kowalik, Daniel Lokshtanov, Dániel Marx, Marcin Pilipczuk, Michal Pilipczuk, and Saket Saurabh. 2015. Parameterized Algorithms. Springer.

[20] Marek Cygan, Stefan Kratsch, and Jesper Nederlof. 2013. Fast hamiltonicity checking via bases of perfect matchings. In Proceedings of the 45th Annual ACM on Symposium on Theory of Computing (STOC'13). 301-310.

[21] Marek Cygan, Jesper Nederlof, Marcin Pilipczuk, Michal Pilipczuk, Johan M. M. van Rooij, and Jakub Onufry Wojtaszczyk. 2011. Solving connectivity problems parameterized by treewidth in single exponential time. In Proceedings of the 52nd Annual IEEE Symposium on Foundations of Computer Science (FOCS'11). 150-159.

[22] Marek Cygan, Marcin Pilipczuk, and Michal Pilipczuk. 2016. Known algorithms for edge clique cover are probably optimal. SIAM f. Comput. 45, 1 (2016), 67-83.

[23] Erik D. Demaine, Fedor V. Fomin, Mohammad Taghi Hajiaghayi, and Dimitrios M. Thilikos. 2005. Subexponential parameterized algorithms on bounded-genus graphs and -minor-free graphs. f. ACM 52, 6 (2005), 866-893.

[24] Erik D. Demaine and MohammadTaghi Hajiaghayi. 2008. The bidimensionality theory and its algorithmic applications. Comput. f. 51, 3 (2008), 292-302.

[25] David Eppstein. 2000. Diameter and treewidth in minor-closed graph families. Algorithmica 27, 3 (2000), $275-291$.

[26] Samuel Fiorini, Nadia Hardy, Bruce A. Reed, and Adrian Vetta. 2008. Planar graph bipartization in linear time. Discrete Appl. Math. 156, 7 (2008).

[27] Jörg Flum and Martin Grohe. 2006. Parameterized Complexity Theory. Springer, Berlin.

[28] Fedor V. Fomin, Serge Gaspers, Saket Saurabh, and Alexey A. Stepanov. 2009. On two techniques of combining branching and treewidth. Algorithmica 54, 2 (2009), 181-207.

[29] Fedor V. Fomin, Petr A. Golovach, Daniel Lokshtanov, and Saket Saurabh. 2014. Almost optimal lower bounds for problems parameterized by clique-width. SIAM J. Comput. 43, 5 (2014), 1541-1563.

[30] Fedor V. Fomin, Daniel Lokshtanov, Fahad Panolan, and Saket Saurabh. 2016. Efficient computation of representative families with applications in parameterized and exact algorithms. F. ACM 63, 4 (2016), 29:1-29:60.

[31] Russell Impagliazzo and Ramamohan Paturi. 2001. On the complexity of k-SAT. f. Comput. Syst. Sci. 62, 2 (2001), 367-375.

[32] Russell Impagliazzo, Ramamohan Paturi, and Francis Zane. 2001. Which problems have strongly exponential complexity? J. Comput. Syst. Sci. 63, 4 (2001), 512-530.

[33] Lars Jaffke and Bart M. P. Jansen. 2017. Fine-grained parameterized complexity analysis of graph coloring problems. In Proceedings of the 10th International Conference on Algorithms and Complexity (CIAC'17) (Lecture Notes in Computer Science), Vol. 10236. 345-356.

[34] Bart M. P. Jansen, Daniel Lokshtanov, and Saket Saurabh. 2014. A near-optimal planarization algorithm. In Proceedings of the 25th Annual ACM-SIAM Symposium on Discrete Algorithms (SODA'14). 1802-1811.

[35] Jon Kleinberg and Éva Tardos. 2005. Algorithm Design. Addison-Wesley Longman Publishing Co., Inc., Boston, MA.

[36] Michael Lampis. 2014. Parameterized approximation schemes using graph widths. In Proceedings of the 41st International Colloquium on Automata, Languages, and Programming (ICALP'14) (Lecture Notes in Computer Science), Vol. 8572. Springer, 775-786.

[37] Daniel Lokshtanov, Dániel Marx, and Saket Saurabh. 2011. Known algorithms on graphs on bounded treewidth are probably optimal. In Proceedings of the 22nd Annual ACM-SIAM Symposium on Discrete Algorithms (SODA'11). 777789.

[38] Dániel Marx. 2007. On the optimality of planar and geometric approximation schemes. In Proceedings of the 48th Annual IEEE Symposium on Foundations of Computer Science (FOCS'07). 338-348.

[39] Dániel Marx. 2010. Can you beat treewidth?Theory Comput. 6, 1 (2010), 85-112.

[40] Dániel Marx and Valia Mitsou. 2016. Double-exponential and triple-exponential bounds for choosability problems parameterized by treewidth. In 43rd International Colloquium on Automata, Languages, and Programming (ICALP'16) (LIPIcs), Vol. 55. 28:1-28:15.

[41] Daniel Mölle, Stefan Richter, and Peter Rossmanith. 2008. Enumerate and expand: Improved algorithms for connected vertex cover and tree cover. Theory Comput. Syst. 43, 2 (2008), 234-253.

[42] Jesper Nederlof, Johan M. M. van Rooij, and Thomas C. van Dijk. 2014. Inclusion/exclusion meets measure and conquer. Algorithmica 69, 3 (2014), 685-740.

[43] Rolf Niedermeier. 2006. Invitation to Fixed-parameter Algorithms. Oxford Lecture Series in Mathematics and its Applications, Vol. 31. Oxford University Press, Oxford.

[44] Mihai Patrascu and Ryan Williams. 2010. On the possibility of faster SAT algorithms. In Proceedings of the 21st Annual ACM-SIAM Symposium on Discrete Algorithms (SODA'10). 1065-1075. 
[45] Liam Roditty and Virginia Vassilevska Williams. 2013. Fast approximation algorithms for the diameter and radius of sparse graphs. In Proceedings of the 45th Annual ACM on Symposium on Theory of Computing (STOC'13). 515-524.

[46] Alexander D. Scott and Gregory B. Sorkin. 2007. Linear-programming design and analysis of fast algorithms for Max 2-CSP. Discrete Optim. 4, 3-4 (2007), 260-287.

[47] Atsushi Takahashi, Shuichi Ueno, and Yoji Kajitani. 1995. Mixed searching and proper-path-width. Theor. Comput. Sci. 137, 2 (1995), 253-268.

[48] Jan Arne Telle and Andrzej Proskurowski. 1993. Practical algorithms on partial k-trees with an application to domination-like problems. In Proceedings of the 3rd Workshop on Algorithms and Data Structures (WADS'93) (Lecture Notes in Computer Science), Vol. 709. Springer, 610-621.

[49] Dimitrios M. Thilikos, Maria J. Serna, and Hans L. Bodlaender. 2005. Cutwidth I: A linear time fixed parameter algorithm. F. Algor. 56, 1 (2005), 1-24.

[50] Johan M. M. van Rooij, Hans L. Bodlaender, and Peter Rossmanith. 2009. Dynamic programming on tree decompositions using generalised fast subset convolution. In Proceedings of the 17th Annual European Symposium on Algorithms (ESA'09) (Lecture Notes in Computer Science), Vol. 5757. Springer, 566-577.

[51] Virginia Vassilevska Williams. 2015. Hardness of easy problems: Basing hardness on popular conjectures such as the strong exponential time hypothesis (invited talk). In Proceedings of the 10th International Symposium on Parameterized and Exact Computation (IPEC'15) (LIPIcs), Vol. 43. 17-29.

Received March 2017; revised October 2017; accepted November 2017 